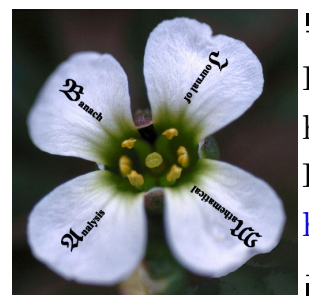

Banach J. Math. Anal. 9 (2015), no. 3, 43-74

http://doi.org/10.15352/bjma/09-3-4

ISSN: $1735-8787$ (electronic)

http://projecteuclid.org/bjma

\title{
ON E-FRAMES IN SEPARABLE HILBERT SPACES
}

\author{
GHOLAMREZA TALEBI*, MOHAMMAD ALI DEHGHAN \\ Communicated by M. Frank
}

\begin{abstract}
The purpose of this paper is to introduce the concept of E-frames for a separable Hilbert space $\mathcal{H}$, where $\mathrm{E}$ is an invertible infinite matrix mapping on the Hilbert space $\underset{n=1}{\infty} \mathcal{H}$. We investigate and study some properties of $\mathrm{E}$-frames and characterize all $\mathrm{E}$-frames for $\mathcal{H}$. Further more, we characterize all dual E-frames associated with a given $\mathrm{E}$-frame. A similar characterization is also established for $\mathrm{E}$-orthonormal bases, E-Riesz bases and dual E-Riesz bases. In continue we obtain a lower estimate for the lower bound of some matrix operators on the $p$-bounded variation sequence space $b v_{p}$ and Euler weighted sequence space $e_{w, p}^{\theta}$. Then we deal with several types of E-frames such as $\boldsymbol{\Delta}$-frames and Euler frames for $\mathcal{H}$ which are related to the Hilbert spaces $b v_{2}$ and $e_{2}^{\theta}$, respectively.
\end{abstract}

Key-Words: E-frame; E-orthonormal basis; E-Bessel sequence; E-Riesz basis; Direct sum of Hilbert spaces; Euler frame; $\boldsymbol{\Delta}$-frame.

\section{INTRODUCTION AND PRELIMINARIES}

Suppose that $X$ and $Y$ be two sequence spaces and $\mathrm{A}=\left(a_{n, k}\right)_{n, k \geq 1}$ be an infinite matrix of real or complex numbers. Then, we say that $A$ defines a matrix mapping from $X$ into $Y$, and we denote it by writing $\mathrm{A}: X \rightarrow Y$, if for every sequence $x=\left\{x_{n}\right\}_{n=1}^{\infty} \in X$, the sequence $A x=\left\{(A x)_{n}\right\}_{n=1}^{\infty}$, the A-transform of

Date: Received: Jul. 29, 2014; Accepted: Sep. 29, 2014.

* Corresponding author.

2010 Mathematics Subject Classification. Primary 42C40; Secondary 47A05, 54D55.

Key words and phrases. E-frame, E-orthonormal basis, E-Bessel sequence, E-Riesz basis, direct sum of Hilbert spaces, Euler frame, $\boldsymbol{\Delta}$-frame. 
$x$, is in $Y$, where

$$
(\mathrm{A} x)_{n}=\sum_{k=1}^{\infty} a_{n, k} x_{k}, n=1,2, \ldots .
$$

Let $\left(\mathcal{H}_{n}\right)_{n=1}^{\infty}$ be a sequence of Hilbert spaces, and let

$$
\bigoplus_{n=1}^{\infty} \mathcal{H}_{n}=\left\{\left(h_{n}\right)_{n=1}^{\infty}: h_{n} \in \mathcal{H}_{n}, \sum_{n=1}^{\infty}\left\|h_{n}\right\|_{\mathcal{H}_{n}}^{2}<\infty\right\} .
$$

Define an inner product $\langle.,$.$\rangle on \bigoplus_{n=1}^{\infty} \mathcal{H}_{n}$ by:

$$
\left\langle\left(g_{n}\right)_{n=1}^{\infty},\left(h_{n}\right)_{n=1}^{\infty}\right\rangle:=\sum_{n=1}^{\infty}\left\langle g_{n}, h_{n}\right\rangle_{\mathcal{H}_{n}} .
$$

With respect to this inner product, $\bigoplus_{n=1}^{\infty} \mathcal{H}_{n}$ is a Hilbert space, called the Hilbert space direct sum of the $\left(\mathcal{H}_{n}\right)_{n=1}^{\infty}[12]$.

Next, let $(\mathcal{H},\langle.,\rangle$.$) be a separable Hilbert space. A countable family \left\{f_{k}\right\}$ in $\mathcal{H}$ is a frame for $\mathcal{H}$ if there exist positive real numbers $A$ and $B$ such that

$$
A\|f\|^{2} \leq\left\|\left\{\left\langle f, f_{k}\right\rangle\right\}_{k=1}^{\infty}\right\|_{\ell^{2}}^{2} \leq B\|f\|^{2}, \forall f \in \mathcal{H} .
$$

The sequence $\left\{f_{k}\right\} \subset \mathcal{H}$ is called a Bessel sequence for $\mathcal{H}$, when it satisfies the upper frame inequality.

In 1952, Duffin and Schaeffer [15] introduced the notion of frame in nonharmonic Fourier analysis. The work of Duffin and Schaeffer was not continued until 1986 when Daubechies, Grossmann and Meyer [13] applied the theory of frame to wavelet and Gabor transform. Frames have very important and interesting properties which make them very useful in the characterization of function spaces, signal processing and many other fields such as image processing, data compressing, sampling theory and so on. A frame for $\mathcal{H}$ is not necessarily a basis for $\mathcal{H}$, but still it implies that every element $f \in \mathcal{H}$ can be represented as $f=\sum c_{k}(f) f_{k}$ for some coefficients $\left\{c_{k}\right\} \in \ell^{2}$ which are not necessarily unique. Theory of frames for Hilbert spaces have been developed deeply. Many people have done momentous works in this field such as Han, Larson, Young, Casazza, Christensen and Cao (see [6, 11, 8, 17, 23]).

In 1990, Grochenig, Aldroubi, Sung and Tang began to study the theory of frames for Banach spaces. They introduced two kinds of notions of frames for Banach spaces: Banach frames and $p$-frames $(1<p<\infty)$. A sequence $\left\{g_{k}\right\}$ in the dual space $\mathrm{X}^{*}$ of a Banach space $\mathrm{X}$ is a $\mathbf{p}$-frame for $\mathrm{X}$ if there exist positive real numbers $A$ and $B$ such that

$$
A\|x\| \leq\left\|\left\{\left\langle x, g_{k}\right\rangle\right\}_{k=1}^{\infty}\right\|_{\ell^{p}} \leq B\|x\|, \quad \forall x \in X .
$$

A Banach frame with respect to $\ell^{p}$ for $\mathrm{X}$ is a $p$-frame for $\mathrm{X}$ with a reconstruction operator $S$ (see[1, 16]). Subsequently, Casazza, Christensen and Stoeva in [7] generalized the concept of $p$-frames and introduce $X_{d}$-frames, where $\mathrm{X}_{d}$ is a $B K$-space. In [18], Li and Cao introduced the notion of $\mathrm{X}_{d}$ frames and $\mathrm{X}_{d}$ Riesz bases for Banach spaces. 
Lately, in [14], the authors introduced the concept of $g$-duals of a frame in a separable Hilbert space $\mathcal{H}$ : A frame $\left\{g_{k}\right\}$ is called a generalized dual frame or a $g$-dual frame of a given frame $\left\{f_{k}\right\}$ for $\mathcal{H}$ if there exists an invertible operator $\mathrm{U} \in B(\mathcal{H})$, such that

$$
f=\sum_{k}\left\langle U f, g_{k}\right\rangle f_{k}, \quad \forall f \in \mathcal{H} .
$$

Moreover, they characterized all generalized dual frames for a given frame.

In 2003, Basar and Altay in [3] introduced and studied the $p$-bounded variation sequence space $b v_{p}$. They proved that this space is linearly isomorphic to the space $\ell^{p}$ and that the space $b v_{2}$ is a separable Hilbert space. Afterward, in 2006, the same authors and Mursaleen in [2] introduced the Euler sequence space $e_{p}^{\theta}$, where $0<\theta<1$. They demonstrated that this space is also linearly isomorphic to the space $\ell^{p}$ and that the space $e_{2}^{\theta}$ is a separable Hilbert space. Here the sequence spaces $b v_{p}$ and $e_{p}^{\theta}$ are defined as

$$
b v_{p}=\left\{\left(x_{n}\right):\left\|\left(x_{n}\right)\right\|_{e_{p}^{\theta}}:=\left\{\sum_{k=1}^{\infty}\left|x_{k}-x_{k-1}\right|^{p}\right\}^{1 / p}<\infty\right\}
$$

and

$$
e_{p}^{\theta}=\left\{\left(x_{n}\right):\left\|\left(x_{n}\right)\right\|_{e_{p}^{\theta}}:=\left\{\sum_{n=1}^{\infty}\left|\sum_{k=1}^{n}\left(\begin{array}{c}
n-1 \\
k-1
\end{array}\right)(1-\theta)^{n-k} \theta^{k-1} x_{k}\right|^{p}\right\}^{1 / p}<\infty\right\},
$$

respectively.

Recently, when we were working on the lower bound problem of some matrix operators on this two sequence spaces, we became interested in studying the sequences $\left\{f_{k}\right\}$, in a Hilbert space $\mathcal{H}$, for which there exist positive real numbers $A$ and $B$ such that

$$
A\|f\| \leq\left\|\left\{\left\langle f, f_{k}\right\rangle\right\}_{k=1}^{\infty}\right\|_{b v_{p}} \leq B\|f\|, \quad \forall f \in \mathcal{H}
$$

or

$$
A\|f\| \leq\left\|\left\{\left\langle f, f_{k}\right\rangle\right\}_{k=1}^{\infty}\right\|_{e_{2}^{\theta}} \leq B\|f\|, \quad \forall f \in \mathcal{H} .
$$

For some reason we named the first $\boldsymbol{\Delta}$-frames and the second Euler frames. This two types of frames motivated us to introduce and study a more general concept of frames namely $\mathrm{E}$-frames for a separable Hilbert space $\mathcal{H}$, where $\mathrm{E}$ is an invertible infinite matrix mapping on the Hilbert space direct sum $\underset{n=1}{\infty} \mathcal{H}$. We investigate some properties of $\mathrm{E}$-frames and characterize all $\mathrm{E}$-frames for $\mathcal{H}$. Our results generalize the concept of frames because the ordinary frames are the special case of $\mathrm{E}$-frames in which the matrix $\mathrm{E}$ be replaced by the identity matrix operator I on $\bigoplus_{n=1}^{\infty} \mathcal{H}$.

The organization of this paper is as follows. In Section 2, we introduce the concepts of E-frames, E-Riesz bases and E-orthonormal bases for an arbitrary separable Hilbert space $\mathcal{H}$. We study some of their properties and characterize them and their duals E-frames. In section 3, we first consider the lower bound problem for some matrix operators on the sequence space $b v_{p}$ and the 
Euler weighted sequence space $e_{w, p}^{\theta}$ and establish a lower estimate for their lower bound. Then we study the concept of $\boldsymbol{\Delta}$-frames and Euler frames as special cases of $\mathrm{E}$-frames for $\mathcal{H}$ which are related to the Hilbert spaces $b v_{2}$ and $e_{2}^{\theta}$, respectively. We compare this two kinds of frames with the ordinary frames, and characterize all $\boldsymbol{\Delta}$-frames, $\boldsymbol{\Delta}$-Riesz bases and $\boldsymbol{\Delta}$-orthonormal bases starting with an arbitrary orthonormal basis for $\mathcal{H}$. A similar characterization is also presented for all Euler frames, Euler Riesz bases and Euler orthonormal bases in $\mathcal{H}$. Moreover, in this section all dual $\boldsymbol{\Delta}$-frames and all dual Euler frames associated with a given $\boldsymbol{\Delta}$-frame and Euler frame, respectively, are identified. Finally, a similar result is also obtained for a new type of E-frames namely Hausdorff frames.

Thought this paper we suppose that $\mathcal{H}$ is a separable Hilbert space and $\mathrm{E}$ is an invertible infinite matrix mapping on the Hilbert space direct sum $\underset{n=1}{\infty} \mathcal{H}$.

\section{E-Frames for separable Hilbert spaces}

Definition 2.1. A sequence $\left\{f_{k}\right\}_{k=1}^{\infty}$ in $\mathcal{H}$ is called an E-Bessel sequence if there exists a constant $B>0$ such that

$$
\left\|\left\{\left\langle f,\left(E\left\{f_{j}\right\}_{j=1}^{\infty}\right)_{k}\right\rangle\right\}_{k=1}^{\infty}\right\|_{\ell^{2}}^{2} \leq B\|f\|^{2}, \forall f \in \mathcal{H}
$$

Any number $B$ satisfying (2.1) is called a E-Bessel bound for $\left\{f_{k}\right\}_{k=1}^{\infty}$. The optimal bound for a given E-Bessel sequence $\left\{f_{k}\right\}_{k=1}^{\infty}$ is the smallest possible value of $B>0$ satisfying (2.1). Inequality (2.1) also can be written as

$$
\sum_{n=1}^{\infty}\left|\left\langle f, \sum_{k=1}^{\infty} E_{n, k} f_{k}\right\rangle\right|^{2} \leq B\|f\|^{2}, \forall f \in \mathcal{H} .
$$

One can easily check that for given constant $B>0$, the sequence $\left\{f_{k}\right\}_{k=1}^{\infty}$ is a $\mathrm{E}$-Bessel sequence with $\mathrm{E}$-Bessel bound $B$ if and only if the operator T defined by

$$
\mathrm{T}: \ell^{2} \rightarrow \mathcal{H}, \quad \mathrm{T}\left\{c_{k}\right\}_{k=1}^{\infty}=\sum_{k=1}^{\infty} c_{k}\left(E\left\{f_{j}\right\}_{j=1}^{\infty}\right)_{k},
$$

is a bounded operator from $\ell^{2}$ into $\mathcal{H}$ with $\|T\| \leq \sqrt{B}$, that its adjoint is given by

$$
\mathrm{T}^{*}: \mathcal{H} \rightarrow \ell^{2}, \mathrm{~T}^{*} f=\left\{\left\langle f,\left(E\left\{f_{j}\right\}_{j=1}^{\infty}\right)_{k}\right\rangle\right\}_{k=1}^{\infty},
$$

and that in this case the inequality (2.1) is satisfied. Thus, if $\left\{f_{k}\right\}_{k=1}^{\infty}$ is a sequence in $\mathcal{H}$ and the sum $\sum_{k=1}^{\infty} c_{k}\left(E\left\{f_{j}\right\}_{j=1}^{\infty}\right)_{k}$ is convergent for all $\left\{c_{k}\right\}_{k=1}^{\infty} \in \ell^{2}$, then $\left\{f_{k}\right\}_{k=1}^{\infty}$ is an E-Bessel sequence. Also, the $\mathrm{E}$-Bessel condition (2.1) remains the same, regardless of how the elements $\left\{f_{k}\right\}_{k=1}^{\infty}$ are numbered. So, for an E-Bessel sequence $\left\{f_{k}\right\}_{k=1}^{\infty}$, the sum $\sum_{k=1}^{\infty} c_{k}\left(E\left\{f_{j}\right\}_{j=1}^{\infty}\right)_{k}$ converges unconditionally for all $\left\{c_{k}\right\}_{k=1}^{\infty} \in \ell^{2}$. Moreover, it is easy verification to see that it is enough to check the Bessel condition (2.1) on a dense subset of $\mathcal{H}$

Definition 2.2. Consider a sequence $\left\{g_{k}\right\}_{k=1}^{\infty}$ of vectors in $\mathcal{H}$. 
(i) The sequence $\left\{g_{k}\right\}_{k=1}^{\infty}$ is an E-complete if $\overline{\operatorname{span}}\left\{\left(E\left\{g_{j}\right\}_{j=1}^{\infty}\right)_{k}\right\}_{k=1}^{\infty}=\mathcal{H}$.

(ii) The sequence $\left\{g_{k}\right\}_{k=1}^{\infty}$ is an E-basis(E-Schauder basis) for $\mathcal{H}$, if for each $f \in \mathcal{H}$ there exist unique scalar coefficients $\left\{c_{k}(f)\right\}_{k=1}^{\infty}$ such that

$$
f=\sum_{k=0}^{\infty} c_{k}(f)\left(E\left\{g_{j}\right\}_{j=1}^{\infty}\right)_{k} .
$$

(iii) An E-basis $\left\{g_{k}\right\}_{k=1}^{\infty}$ is unconditional, if the series (2.2) converges unconditionally for each $f \in \mathcal{H}$.

(iv) An E-basis $\left\{g_{k}\right\}_{k=1}^{\infty}$ is E-orthonormal, if $\left\{g_{k}\right\}_{k=1}^{\infty}$ is an E-orthonormal system, i.e., if

$$
\left\langle\left(E\left\{g_{j}\right\}_{j=1}^{\infty}\right)_{n},\left(E\left\{g_{j}\right\}_{j=1}^{\infty}\right)_{k}\right\rangle=\delta_{n, k}= \begin{cases}1 & n=k \\ 0 & n \neq k\end{cases}
$$

It is an easy verification to see that every separable Hilbert space $\mathcal{H}$ has an E-orthonormal basis and that every E-orthonormal basis is an E-Bessl sequence. In fact, if $\left\{g_{k}\right\}_{k=1}^{\infty}$ is an E-orthonormal basis for $\mathcal{H}$ and $\left\{c_{k}\right\}_{k=1}^{\infty} \in \ell^{2}$, then

$$
\left\|\sum_{k=1}^{\infty} c_{k}\left(E\left\{f_{j}\right\}_{j=1}^{\infty}\right)_{k}\right\|^{2}=\sum_{k=1}^{\infty}\left|c_{k}\right|^{2},
$$

which implies that $\left\{g_{k}\right\}_{k=1}^{\infty}$ is an E-Bessl sequence with E-Bessl bound 1. Also, Obviously every E-orthonormal basis is E-complete.

The next theorem gives equivalent conditions for an E-orthonormal system $\left\{g_{k}\right\}_{k=1}^{\infty}$ to be an E-orthonormal basis. Its proof can be easily adapted to one of ([11], Theorem 3.2.2).

Theorem 2.3. For a E-orthonormal system $\left\{g_{k}\right\}_{k=1}^{\infty}$, the following are equivalent:

(i) $\left\{g_{k}\right\}_{k=1}^{\infty}$ is an E-orthonormal basis.

(ii) $f=\sum_{k=1}^{\infty}\left\langle f,\left(E\left\{g_{j}\right\}_{j=1}^{\infty}\right)_{k}\right\rangle\left(E\left\{g_{j}\right\}_{j=1}^{\infty}\right)_{k}, \forall f \in \mathcal{H}$.

(iii) $\langle f, g\rangle=\sum_{k=1}^{\infty}\left\langle f,\left(E\left\{g_{j}\right\}_{j=1}^{\infty}\right)_{k}\right\rangle\left\langle\left(E\left\{g_{j}\right\}_{j=1}^{\infty}\right)_{k}, g\right\rangle, \forall f, g \in \mathcal{H}$.

(iv) $\left\|\left\{\left\langle f,\left(E\left\{g_{j}\right\}_{j=1}^{\infty}\right)_{k}\right\rangle\right\}_{k=1}^{\infty}\right\|_{\ell^{2}}^{2}=\|f\|^{2}, \quad \forall f \in \mathcal{H}$.

(v) $\left\{g_{k}\right\}_{k=1}^{\infty}$ is an $\mathrm{E}$-complete sequence.

(vi) If $\left\langle f,\left(E\left\{g_{j}\right\}_{j=1}^{\infty}\right)_{k}\right\rangle=0, \forall k \in \mathbb{N}$, then $f=0$.

The following theorem characterizes all E-orthonormal bases for $\mathcal{H}$ starting with one arbitrary orthonormal basis. 
Theorem 2.4. Let $\left\{e_{k}\right\}_{k=1}^{\infty}$ be an orthonormal basis for $\mathcal{H}$. Then the $\mathrm{E}$-orthonormal bases for $\mathcal{H}$ are precisely the sets $\left\{U\left(E^{-1}\left\{e_{j}\right\}_{j=1}^{\infty}\right)_{k}\right\}_{k=1}^{\infty}$, where $U$ is an unitary operator on $\mathcal{H}$.

Proof. Let $\left\{f_{k}\right\}_{k=1}^{\infty}$ be an E-orthonormal basis for $\mathcal{H}$. Define the operator

$$
U: \mathcal{H} \rightarrow \mathcal{H}, \quad U\left(\sum c_{k} e_{k}\right)=\sum c_{k}\left(E\left\{f_{j}\right\}_{j=1}^{\infty}\right)_{k},\left\{c_{k}\right\}_{k=1}^{\infty} \in \ell^{2} .
$$

Then $U$ maps $\mathcal{H}$ boundedly and bijectively onto $\mathcal{H}$, and we have $f_{k}=U\left(E^{-1}\left\{e_{j}\right\}_{j=1}^{\infty}\right)_{k}$. For $f, g \in \mathcal{H}$, write $f=\sum\left\langle f, e_{k}\right\rangle e_{k}$ and $g=\sum\left\langle g, e_{k}\right\rangle e_{k}$; then, via the definition of $U$, we have

$$
\begin{aligned}
\left\langle U^{*} U f, g\right\rangle & =\langle U f, U g\rangle \\
& =\left\langle\sum\left\langle f, e_{k}\right\rangle\left(E\left\{f_{j}\right\}_{j=1}^{\infty}\right)_{k}, \sum\left\langle g, e_{k}\right\rangle\left(E\left\{f_{j}\right\}_{j=1}^{\infty}\right)_{k}\right\rangle \\
& =\sum\left\langle f, e_{k}\right\rangle \overline{\left\langle g, e_{k}\right\rangle} \\
& =\langle f, g\rangle,
\end{aligned}
$$

which implies that $U^{*} U=I$, and since $U$ is surjective, it follows that $U$ is unitary. On the other hand, if $U$ is a given unitary operator, then

$$
\begin{aligned}
\left\langle\left(E\left\{U\left(E^{-1}\left\{e_{i}\right\}_{i=1}^{\infty}\right)_{j}\right\}_{j=1}^{\infty}\right)_{n},\left(E\left\{U\left(E^{-1}\left\{e_{i}\right\}_{i=1}^{\infty}\right)_{j}\right\}_{j=1}^{\infty}\right)_{k}\right\rangle \\
=\left\langle U\left(E\left\{\left(E^{-1}\left\{e_{i}\right\}_{i=1}^{\infty}\right)_{j}\right\}_{j=1}^{\infty}\right)_{n}, U\left(E\left\{\left(E^{-1}\left\{e_{i}\right\}_{i=1}^{\infty}\right)_{j}\right\}_{j=1}^{\infty}\right)_{k}\right\rangle \\
=\left\langle U^{*} U\left(E\left\{\left(E^{-1}\left\{e_{i}\right\}_{i=1}^{\infty}\right)_{j}\right\}_{j=1}^{\infty}\right)_{n},\left(E\left\{\left(E^{-1}\left\{e_{i}\right\}_{i=1}^{\infty}\right)_{j}\right\}_{j=1}^{\infty}\right)_{k}\right\rangle \\
=\left\langle\left(E\left\{\left(E^{-1}\left\{e_{i}\right\}_{i=1}^{\infty}\right)_{j}\right\}_{j=1}^{\infty}\right)_{n},\left(E\left\{\left(E^{-1}\left\{e_{i}\right\}_{i=1}^{\infty}\right)_{j}\right\}_{j=1}^{\infty}\right)_{k}\right\rangle_{k} \\
=\left\langle e_{n}, e_{k}\right\rangle=\delta_{n, k} .
\end{aligned}
$$

i.e., $\left\{U\left(E^{-1}\left\{e_{j}\right\}_{j=1}^{\infty}\right)_{k}\right\}_{k=1}^{\infty}$ is an E-orthonormal system. Also, we have

$$
\begin{aligned}
\left\|\left\{\left\langle f, U\left(E^{-1}\left\{e_{i}\right\}_{i=1}^{\infty}\right)_{j}\right\rangle\right\}_{j=1}^{\infty}\right\|_{\ell^{2}}^{2} & =\sum_{n=1}^{\infty}\left|\left\langle f,\left(E\left\{U\left(E^{-1}\left\{e_{i}\right\}_{i=1}^{\infty}\right)_{j}\right\}_{j=1}^{\infty}\right){ }_{n}\right\rangle\right|^{2} \\
& =\sum_{n=1}^{\infty}\left|\left\langle f, U\left(E\left\{\left(E^{-1}\left\{e_{i}\right\}_{i=1}^{\infty}\right)_{j}\right\}_{j=1}^{\infty}\right){ }_{n}\right\rangle\right|^{2} \\
& =\sum_{n=1}^{\infty}\left|\left\langle U^{*} f, e_{n}\right\rangle\right|^{2}=\left\|U^{*} f\right\|^{2} .
\end{aligned}
$$

Now, it follows from Theorem 2.3 that the sequence $\left\{U\left(E^{-1}\left\{e_{j}\right\}_{j=1}^{\infty}\right)_{k}\right\}_{k=1}^{\infty}$ is an E-orthonormal basis. This completes the proof. 
Without assuming that $\left\{g_{k}\right\}_{k=1}^{\infty}$ is an E-orthonormal system, it implies that $\left\{g_{k}\right\}_{k=1}^{\infty}$ is an E-orthonormal basis if the vectors $\left\{\left(E\left\{g_{j}\right\}_{j=1}^{\infty}\right)_{k}\right\}_{k=1}^{\infty}$ are normalized.

Proposition 2.5. Assume that $\left\{g_{k}\right\}_{k=1}^{\infty}$ is a sequence of vectors in $\mathcal{H}$ such that $\left\|\left(E\left\{g_{j}\right\}_{j=1}^{\infty}\right)_{k}\right\|=1$, for all $k$ and that

$$
\left\|\left\{\left\langle f,\left(E\left\{g_{j}\right\}_{j=1}^{\infty}\right)_{k}\right\rangle\right\}_{k=1}^{\infty}\right\|_{\ell^{2}}^{2}=\|f\|^{2}, \quad \forall f \in \mathcal{H}
$$

Then $\left\{g_{k}\right\}_{k=1}^{\infty}$ is an $\mathrm{E}$-orthonormal basis for $\mathcal{H}$.

In Theorem 2.4, we have characterized all E-orthonormal bases in terms of unitary operators acting on a single orthonormal basis. Formally, the definition of an E-Riesz basis appears by weakening the condition on the operator:

Definition 2.6. Let $\left\{e_{k}\right\}_{k=1}^{\infty}$ is an orthonormal basis for $\mathcal{H}$. An E-Riesz basis for $\mathcal{H}$ is a family of the form $\left\{U\left(E^{-1}\left\{e_{j}\right\}_{j=1}^{\infty}\right)_{k}\right\}_{k=1}^{\infty}$, where $U$ is a bounded bijective operator on $\mathcal{H}$.

Theorem 2.7. Let $\left\{f_{k}\right\}_{k=1}^{\infty}$ be an E-Riesz basis for $\mathcal{H}$, then $\left\{f_{k}\right\}_{k=1}^{\infty}$ is an E-Bessel sequence. Furthermore, there exists a unique sequence $\left\{g_{k}\right\}_{k=1}^{\infty}$ in $\mathcal{H}$ such that

$$
f=\sum_{k=1}^{\infty}\left\langle f,\left(E\left\{g_{j}\right\}_{j=1}^{\infty}\right)_{k}\right\rangle\left(E\left\{f_{j}\right\}_{j=1}^{\infty}\right)_{k}, \forall f \in \mathcal{H}
$$

The sequence $\left\{g_{k}\right\}_{k=1}^{\infty}$ is also an $\mathrm{E}$-Riesz basis, and the series (2.3) converges unconditionally for all $f \in \mathcal{H}$.

Proof. According to the Definition 2.6, $\left\{f_{k}\right\}_{k=1}^{\infty}=\left\{U\left(E^{-1}\left\{e_{j}\right\}_{j=1}^{\infty}\right)_{k}\right\}_{k=1}^{\infty}$, in which $U$ is a bounded bijective operator on $\mathcal{H}$ and $\left\{e_{k}\right\}_{k=1}^{\infty}$ is an orthonormal basis for $\mathcal{H}$. By expanding $U^{-1} f$ in terms of the sequence $\left\{e_{k}\right\}_{k=1}^{\infty}$, we have

$$
U^{-1} f=\sum_{k=1}^{\infty}\left\langle U^{-1} f, e_{k}\right\rangle e_{k}=\sum_{k=1}^{\infty}\left\langle f,\left(U^{-1}\right)^{*} e_{k}\right\rangle e_{k}
$$


Therefore

$$
\begin{aligned}
f & =\sum_{k=1}^{\infty}\left\langle U^{-1} f, e_{k}\right\rangle U e_{k}=\sum_{k=1}^{\infty}\left\langle f,\left(U^{-1}\right)^{*} e_{k}\right\rangle U e_{k} \\
& =\sum_{k=1}^{\infty}\left\langle f,\left(U^{-1}\right)^{*}\left(E\left\{\left(E^{-1}\left\{e_{i}\right\}_{i=1}^{\infty}\right)_{j}\right\}_{j=1}^{\infty}\right)_{k}\right\rangle U\left(E\left\{\left(E^{-1}\left\{e_{i}\right\}_{i=1}^{\infty}\right)_{j}\right\}_{j=1}^{\infty}\right)_{k} \\
& =\sum_{k=1}^{\infty}\left\langle f,\left(E\left(U^{-1}\right)^{*}\left\{\left(E^{-1}\left\{e_{i}\right\}_{i=1}^{\infty}\right)_{j}\right\}_{j=1}^{\infty}\right)_{k}\right\rangle\left(E U\left\{\left(E^{-1}\left\{e_{i}\right\}_{i=1}^{\infty}\right)_{j}\right\}_{j=1}^{\infty}\right)_{k} \\
& =\sum_{k=1}^{\infty}\left\langle f,\left(E\left\{\left(U^{-1}\right)^{*}\left(E^{-1}\left\{e_{i}\right\}_{i=1}^{\infty}\right)_{j}\right\}_{j=1}^{\infty}\right)_{k}\right\rangle\left(E\left\{U\left(E^{-1}\left\{e_{i}\right\}_{i=1}^{\infty}\right)_{j}\right\}_{j=1}^{\infty}\right)_{k} \\
& =\sum_{k=1}^{\infty}\left\langle f,\left(E\left\{g_{j}\right\}_{j=1}^{\infty}\right)_{k}\right\rangle\left(E\left\{f_{j}\right\}_{j=1}^{\infty}\right)_{k}
\end{aligned}
$$

where $g_{k}=\left(U^{-1}\right)^{*}\left(E^{-1}\left\{e_{i}\right\}_{i=1}^{\infty}\right)_{k}$. Since the operator $\left(U^{-1}\right)^{*}$ is also bounded and bijective, $\left\{g_{k}\right\}_{k=1}^{\infty}$ is an E-Riesz basis. For $f \in \mathcal{H}$, we have

$$
\begin{aligned}
\sum_{k=1}^{\infty}\left|\left\langle f,\left(E\left\{f_{j}\right\}_{j=1}^{\infty}\right)_{k}\right\rangle\right|^{2} & =\sum_{k=1}^{\infty}\left|\left\langle f,\left(E\left\{U\left(E^{-1}\left\{e_{i}\right\}_{i=1}^{\infty}\right)_{j}\right\}_{j=1}^{\infty}\right)_{k}\right\rangle\right|^{2} \\
& =\sum_{k=1}^{\infty}\left|\left\langle f,\left(U E\left\{\left(E^{-1}\left\{e_{i}\right\}_{i=1}^{\infty}\right)_{j}\right\}_{j=1}^{\infty}\right)_{k}\right\rangle\right|^{2} \\
& =\sum_{k=1}^{\infty}\left|\left\langle f, U\left(E\left\{\left(E^{-1}\left\{e_{i}\right\}_{i=1}^{\infty}\right)_{j}\right\}_{j=1}^{\infty}\right)_{k}\right\rangle\right|^{2} \\
& =\sum_{k=1}^{\infty}\left|\left\langle f, U e_{k}\right\rangle\right|^{2}=\left\|U^{*} f\right\|^{2} \leq\left\|U^{*}\right\|^{2}\|f\|^{2} .
\end{aligned}
$$

This proves that $\left\{f_{k}\right\}_{k=1}^{\infty}$ and also $\left\{g_{k}\right\}_{k=1}^{\infty}$ are E-Bessel sequences. Thus, the series (2.3) converges unconditionally. Showing that the sequence $\left\{g_{k}\right\}_{k=1}^{\infty}$, constructed in the proof, is the only one that satisfies (2.3), is a routine verification and so we omit the details.

The unique sequence $\left\{g_{k}\right\}_{k=1}^{\infty}$ satisfying (2.3) is called the dual E-Riesz basis of $\left\{f_{k}\right\}_{k=1}^{\infty}$. One can easily check that the dual E-Riesz basis of $\left\{g_{k}\right\}_{k=1}^{\infty}$ is $\left\{f_{k}\right\}_{k=1}^{\infty}$. That is $\left\{f_{k}\right\}_{k=1}^{\infty}$ and $\left\{g_{k}\right\}_{k=1}^{\infty}$ are the dual E-Riesz basis of each other. For this reason, we frequently speak about a pair of dual E-Riesz bases. In particular, this implies a symmetric version of (2.3), see (2.5). Furthermore, an E-Riesz basis and its dual E-Riesz basis satisfy an important orthogonality relationship. We state the result right after the following definition.

Definition 2.8. Two sequences $\left\{f_{k}\right\}_{k=1}^{\infty}$ and $\left\{g_{k}\right\}_{k=1}^{\infty}$ in a Hilbert space are E-biorthogonal if

$$
\left\langle\left(E\left\{f_{j}\right\}_{j=1}^{\infty}\right)_{k},\left(E\left\{g_{j}\right\}_{j=1}^{\infty}\right)_{n}\right\rangle=\delta_{k, n}
$$


Corollary 2.9. For a pair of dual E-Riesz bases $\left\{f_{k}\right\}_{k=1}^{\infty}$ and $\left\{g_{k}\right\}_{k=1}^{\infty}$ the following holds:

(i) $\left\{f_{k}\right\}_{k=1}^{\infty}$ and $\left\{g_{k}\right\}_{k=1}^{\infty}$ are E-biorthogonal.

(ii) For all $f \in \mathcal{H}$,

$$
f=\sum_{k=1}^{\infty}\left\langle f,\left(E\left\{g_{j}\right\}_{j=1}^{\infty}\right)_{k}\right\rangle\left(E\left\{f_{j}\right\}_{j=1}^{\infty}\right)_{k}=\sum_{k=1}^{\infty}\left\langle f,\left(E\left\{f_{j}\right\}_{j=1}^{\infty}\right)_{k}\right\rangle\left(E\left\{g_{j}\right\}_{j=1}^{\infty}\right)_{k} .
$$

Proposition 2.10. If $\left\{f_{k}\right\}_{k=1}^{\infty}=\left\{U\left(E^{-1}\left\{e_{i}\right\}_{i=1}^{\infty}\right)_{k}\right\}_{k=1}^{\infty}$ is an E-Riesz basis for $\mathcal{H}$, there exist real positive constants $A$ and $B$ such that

$$
A\|f\|^{2} \leq\left\|\left\{\left\langle f,\left(E\left\{f_{j}\right\}_{j=1}^{\infty}\right)_{k}\right\rangle\right\}_{k=1}^{\infty}\right\|_{\ell^{2}}^{2} \leq B\|f\|^{2}, \quad \forall f \in \mathcal{H} .
$$

The largest possible value for the constant $A$ is $\left\|U^{-1}\right\|^{-2}$, and the smallest possible value for $B$ is $\|U\|^{2}$.

Proof. That an E-Riesz basis $\left\{U\left(E^{-1}\left\{e_{i}\right\}_{i=1}^{\infty}\right)_{k}\right\}_{k=1}^{\infty}$ is an E-Bessel sequence with optimal upper bound $\|U\|^{2}$ follows already from the estimate in (2.4). The result about the lower bound is a consequence of the following calculation

$$
\begin{aligned}
\|f\|^{2}=\left\|\left(U^{*}\right)^{-1} U^{*} f\right\|^{2} & \leq\left\|\left(U^{*}\right)^{-1}\right\|^{2}\left\|U^{*} f\right\|^{2} \\
& =\left\|U^{-1}\right\|^{2}\left\|U^{*} f\right\|^{2}=\left\|U^{-1}\right\|^{2} \sum_{k=1}^{\infty}\left|\left\langle f,\left(E\left\{f_{j}\right\}_{j=1}^{\infty}\right)_{k}\right\rangle\right|^{2}
\end{aligned}
$$

where the last equality is obtained in the same way as (2.4).

It is easy to see that a sequence $\left\{f_{k}\right\}_{k=1}^{\infty}$ in $\mathcal{H}$, is an E-Riesz basis if and only if it is $\mathrm{E}$-complete in $\mathcal{H}$, and there exist real positive constants $A$ and $B$ such that for every finite scalar sequence $\left\{c_{k}\right\}$, one has

$$
A \sum\left|c_{k}\right|^{2} \leq\left\|\sum c_{k}\left(E\left\{f_{j}\right\}_{j=1}^{\infty}\right)_{k}\right\|^{2} \leq B \sum\left|c_{k}\right|^{2}
$$

If (2.6) holds for all finite scalar sequences $\left\{c_{k}\right\}$, then it automatically holds for all $\left\{c_{k}\right\}_{k=1}^{\infty} \in \ell^{2}$. For an E-Riesz basis $\left\{f_{k}\right\}_{k=1}^{\infty}$, the numbers $A$ and $B$ that satisfy (2.6) are called lower and upper E-Riesz bounds, respectively. They are not unique, and we define the optimal E-Riesz bounds as the largest possible value for $A$ and the smallest possible value for $B$. A sequence $\left\{f_{k}\right\}_{k=1}^{\infty}$ satisfying (2.6) for all finite sequences $\left\{c_{k}\right\}_{k=1}^{\infty}$ is called an E-Riesz sequence. Therefore, an E-Riesz sequence $\left\{f_{k}\right\}_{k=1}^{\infty}$ is an E-Riesz basis for $\overline{\operatorname{span}}\left\{\left(E\left\{f_{j}\right\}_{j=1}^{\infty}\right)_{k}\right\}_{k=1}^{\infty}$ and every subfamily of an E-Riesz sequence is an E-Riesz sequence.

Proposition 2.11. Let $\left\{f_{k}\right\}_{k=1}^{\infty}$ be an $\mathrm{E}$-complete sequence in $\mathcal{H}$ and

$$
\left\|\sum_{k=1}^{\infty} c_{k}\left(E\left\{f_{j}\right\}_{j=1}^{\infty}\right)_{k}\right\|^{2}=\sum_{k=1}^{\infty}\left|c_{k}\right|^{2},
$$

for all finite scalar sequences $\left\{c_{k}\right\}$. Then $\left\{f_{k}\right\}_{k=1}^{\infty}$ is an E-orthonormal basis for $\mathcal{H}$. 
Proof. The assumptions imply that $\left\{f_{k}\right\}_{k=1}^{\infty}$ is an E-Reisz basis for $\mathcal{H}$ with bounds $A=B=1$, so we can write $\left\{f_{k}\right\}_{k=1}^{\infty}=\left\{U\left(E^{-1}\left\{e_{i}\right\}_{i=1}^{\infty}\right)_{k}\right\}_{k=1}^{\infty}$ for an appropriate bounded invertible operator $U$, where $\left\{e_{k}\right\}_{k=1}^{\infty}$ is an orthonormal basis for $\mathcal{H}$. Then for all $\left\{c_{k}\right\}_{k=1}^{\infty} \in \ell^{2}$,

$$
\begin{aligned}
\sum_{k=1}^{\infty}\left|c_{k}\right|^{2}=\left\|\sum_{k=1}^{\infty} c_{k}\left(E\left\{f_{j}\right\}_{j=1}^{\infty}\right)_{k}\right\|^{2} & =\left\|\sum_{k=1}^{\infty} c_{k}\left(E\left\{U\left(E^{-1}\left\{e_{i}\right\}_{i=1}^{\infty}\right)_{j}\right\}_{j=1}^{\infty}\right)_{k}\right\|^{2} \\
& =\left\|\sum_{k=1}^{\infty} c_{k} U\left(E\left\{\left(E^{-1}\left\{e_{i}\right\}_{i=1}^{\infty}\right)_{j}\right\}_{j=1}^{\infty}\right)_{k}\right\|^{2} \\
& =\left\|U\left(\sum_{k=1}^{\infty} c_{k} e_{k}\right)\right\|^{2} .
\end{aligned}
$$

It follows from here that $\|U\|=\left\|U^{-1}\right\|=1$; by Proposition 2.10, we conclude that

$$
\left\|\left\{\left\langle f,\left(E\left\{f_{j}\right\}_{j=1}^{\infty}\right)_{k}\right\rangle\right\}_{k=1}^{\infty}\right\|_{\ell^{2}}^{2}=\|f\|^{2}, \quad \forall f \in H .
$$

The assumptions also imply that $\left\|\left(E\left\{f_{j}\right\}_{j=1}^{\infty}\right)_{k}\right\|=1$, for all $k$. The result now obtained via Proposition 2.5.

The Gram matrix G associated with an E-Bessel sequence $\left\{f_{k}\right\}_{k=1}^{\infty}$ which is the combination of the operator $\mathrm{T}$ and its adjoint $\mathrm{T}^{*}$, is defined by

$$
\mathrm{G}:=\mathrm{TT}^{*}=\left\{\left\langle\left(E\left\{f_{j}\right\}_{j=1}^{\infty}\right)_{n},\left(E\left\{f_{j}\right\}_{j=1}^{\infty}\right)_{k}\right\rangle\right\}_{n, k=1}^{\infty}
$$

The conditions for a sequence $\left\{f_{k}\right\}_{k=1}^{\infty}$ being an E-Bessel sequence can conveniently be expressed in terms of its associated Gram matrix. As the same as in ordinary Bessel sequences, a sequence $\left\{f_{k}\right\}_{k=1}^{\infty}$ in $\mathcal{H}$ is an E-Bessel sequence if and only if its Gram matrix defines a bounded operator on $\ell^{2}$.

An application of Schur's lemma, (see [11], Lemma 3.4.2), implies that the Gram matrix is bounded if all whose rows or columns belong to $\ell^{1}$. In the following we present a sufficient condition for a sequence $\left\{f_{k}\right\}_{k=1}^{\infty}$ being an E-Bessel sequence. Its proof is similar to ([11], Proposition 3.4.3).

Proposition 2.12. Consider the sequence $\left\{f_{k}\right\}_{k=1}^{\infty}$ in $\mathcal{H}$ and suppose that there exists a constant $B>0$ such that

$$
\sum_{k=1}^{\infty}\left|\left\langle\left(E\left\{f_{j}\right\}_{j=1}^{\infty}\right)_{n},\left(E\left\{f_{j}\right\}_{j=1}^{\infty}\right)_{k}\right\rangle\right| \leq B, \quad \forall n \in \mathbb{N} .
$$

Then $\left\{f_{k}\right\}_{k=1}^{\infty}$ is an $\mathrm{E}$-Bessel sequence with $\mathrm{E}$-Bessel bound B.

We will now present a further equivalent condition for a sequence $\left\{f_{k}\right\}_{k=1}^{\infty}$ being an E-Riesz basis, expressed in terms of the Gram matrix.

Theorem 2.13. For a sequence $\left\{f_{k}\right\}_{k=1}^{\infty}$ in $\mathcal{H}$, the following conditions are equivalent.

(i) The sequence $\left\{f_{k}\right\}_{k=1}^{\infty}$ is an $\mathrm{E}$-Riesz basis for $\mathcal{H}$. 
(ii) $\left\{f_{k}\right\}_{k=1}^{\infty}$ is E-complete and its Gram matrix $\left\{\left\langle\left(E\left\{f_{j}\right\}_{j=1}^{\infty}\right)_{n},\left(E\left\{f_{j}\right\}_{j=1}^{\infty}\right)_{k}\right\rangle\right\}_{n, k=1}^{\infty}$ defines a bounded, invertible operator on $\ell^{2}$.

(iii) $\left\{f_{k}\right\}_{k=1}^{\infty}$ is an $\mathrm{E}$-complete $\mathrm{E}$-Bessel sequence, and there exists an $\mathrm{E}-$ complete $\mathrm{E}-$ Bessel sequence $\left\{g_{k}\right\}_{k=1}^{\infty}$ which is $\mathrm{E}$-biorthogonal with $\left\{f_{k}\right\}_{k=1}^{\infty}$.

Proof. Write $\left\{f_{k}\right\}_{k=1}^{\infty}=\left\{U\left(E^{-1}\left\{e_{j}\right\}_{j=1}^{\infty}\right)_{k}\right\}_{k=1}^{\infty}$ as in the definition of E-Riesz basis. For any $n, k \in \mathbb{N}$,

$$
\begin{aligned}
\left\langle\left(E\left\{f_{j}\right\}_{j=1}^{\infty}\right)_{n},\left(E\left\{f_{j}\right\}_{j=1}^{\infty}\right)_{k}\right\rangle & \\
& =\left\langle\left(E\left\{U\left(E^{-1}\left\{e_{i}\right\}_{i=1}^{\infty}\right)_{j}\right\}_{j=1}^{\infty}\right)_{n},\left(E\left\{U\left(E^{-1}\left\{e_{i}\right\}_{i=1}^{\infty}\right)_{j}\right\}_{j=1}^{\infty}\right)_{k}\right\rangle \\
& =\left\langle U\left(E\left\{\left(E^{-1}\left\{e_{i}\right\}_{i=1}^{\infty}\right)_{j}\right\}_{j=1}^{\infty}\right)_{n}, U\left(E\left\{\left(E^{-1}\left\{e_{i}\right\}_{i=1}^{\infty}\right)_{j}\right\}_{j=1}^{\infty}\right)_{k}\right\rangle \\
& =\left\langle U e_{n}, U e_{k}\right\rangle=\left\langle U^{*} U e_{n}, e_{k}\right\rangle,
\end{aligned}
$$

i.e., the Gram matrix is the matrix representing the bounded invertible operator $U^{*} U$ in the basis $\left\{e_{k}\right\}_{k=1}^{\infty}$.

(ii) $\Rightarrow$ (i): It is enough to show the left hand side inequality in (2.6). Given a sequence $\left\{c_{k}\right\}_{k=1}^{\infty} \in \ell^{2}$, we have

$$
\begin{aligned}
\left\langle G\left\{c_{k}\right\}_{k=1}^{\infty},\left\{c_{k}\right\}_{k=1}^{\infty}\right\rangle & =\sum_{k=1}^{\infty} \sum_{n=1}^{\infty}\left\langle\left(E\left\{f_{j}\right\}_{j=1}^{\infty}\right)_{n},\left(E\left\{f_{j}\right\}_{j=1}^{\infty}\right)_{k}\right\rangle c_{n} \overline{c_{k}} \\
& =\left\|\sum_{k=1}^{\infty} c_{k}\left(E\left\{f_{j}\right\}_{j=1}^{\infty}\right)_{k}\right\|^{2} .
\end{aligned}
$$

Thus $\mathrm{G}$ is positive. A similar calculation shows that $\mathrm{G}$ is self-adjoint and therefore has a positive square-root $\mathrm{V}$. By the above calculation

$$
\left\|\sum_{k=1}^{\infty} c_{k}\left(E\left\{f_{j}\right\}_{j=1}^{\infty}\right)_{k}\right\|^{2}=\left\|V\left\{c_{k}\right\}_{k=1}^{\infty}\right\|^{2} \geq \frac{1}{\left\|V^{-1}\right\|^{2}} \sum_{k=1}^{\infty}\left|c_{k}\right|^{2} .
$$

Hence the left hand side inequality in (2.6) is obtained. (i) $\Rightarrow$ (iii): An E-Riesz basis is clearly E-complete. Now, Corollary 2.9 shows that the E-Riesz basis and its dual E-Riesz basis form an E-biorthogonal system and both are E-Bessel sequences.

(iii) $\Rightarrow$ (i): Letting $\left\{e_{k}\right\}_{k=1}^{\infty}$ be an orthonormal basis for $\mathcal{H}$, we can define an operator

$$
V: \operatorname{span}\left\{\left(E\left\{f_{j}\right\}_{j=1}^{\infty}\right)_{k}\right\}_{k=1}^{\infty} \rightarrow \mathcal{H}, \quad V \sum c_{k}\left(E\left\{f_{j}\right\}_{j=1}^{\infty}\right)_{k}=\sum c_{k} e_{k} .
$$


Writing $f \in \operatorname{span}\left\{\left(E\left\{f_{j}\right\}_{j=1}^{\infty}\right)_{k}\right\}_{k=1}^{\infty}$ as $f=\sum\left\langle f,\left(E\left\{g_{j}\right\}_{j=1}^{\infty}\right)_{k}\right\rangle\left(E\left\{f_{j}\right\}_{j=1}^{\infty}\right)_{k}$, and letting $C$ denote an E-Bessel bound for $\left\{g_{k}\right\}_{k=1}^{\infty}$, we have

$$
\|V f\|^{2}=\left\|\sum\left\langle f,\left(E\left\{g_{j}\right\}_{j=1}^{\infty}\right)_{k}\right\rangle e_{k}\right\|^{2}=\sum\left|\left\langle f,\left(E\left\{g_{j}\right\}_{j=1}^{\infty}\right)_{k}\right\rangle\right|^{2} \leq C\|f\|^{2} .
$$

By E-completeness of $\left\{f_{k}\right\}_{k=1}^{\infty}, \mathrm{V}$ has an extension to a bounded operator on $\mathcal{H}$. By the same manner we can extend the linear map

$$
W: \operatorname{span}\left\{\left(E\left\{g_{j}\right\}_{j=1}^{\infty}\right)_{k}\right\}_{k=1}^{\infty} \rightarrow \mathcal{H}, \quad W \sum c_{k}\left(E\left\{g_{j}\right\}_{j=1}^{\infty}\right)_{k}=\sum c_{k} e_{k}
$$

to a bounded operator on $\mathcal{H}$. From the assumption and via an easy calculation, we deduce

$$
\langle W f, V g\rangle=\langle f, g\rangle, \quad \forall f, g \in \mathcal{H}
$$

which implies that for any $h \in \mathcal{H}$,

$$
\|h\|^{2}=\langle h, h\rangle=\langle W h, V h\rangle \leq\|V h\|\|W\|\|h\| .
$$

It follows that $\mathrm{V}$ is injective. $\mathrm{V}$ is also surjective: in fact, given $g \in \mathcal{H}$, we can write $g=\sum_{k=1}^{\infty}\left\langle g, e_{k}\right\rangle e_{k}=V\left(\sum_{k=1}^{\infty}\left\langle g, e_{k}\right\rangle\left(E\left\{f_{j}\right\}_{j=1}^{\infty}\right)_{k}\right)$. Since $f_{k}=$ $V^{-1}\left(E^{-1}\left\{e_{i}\right\}_{i=1}^{\infty}\right)_{k}$, we conclude that $\left\{f_{k}\right\}_{k=1}^{\infty}$ is an E-Riesz basis. The proof is now completed.

We have the following characterization of the optimal E-Riesz bounds in terms of the operators appearing in the proof of Theorem 2.13.

Corollary 2.14. Let $\left\{f_{k}\right\}_{k=1}^{\infty}=\left\{U\left(E^{-1}\left\{e_{i}\right\}_{i=1}^{\infty}\right)_{k}\right\}_{k=1}^{\infty}$ be an E-Riesz basis for $\mathcal{H}$, and $\mathrm{G}: \ell^{2} \rightarrow \ell^{2}$ be its associated Gram matrix. Then the optimal E-Riesz bounds are

$$
A=\frac{1}{\left\|U^{-1}\right\|^{2}}=\frac{1}{\left\|G^{-1}\right\|} \quad \& \quad B=\|U\|^{2}=\|G\| .
$$

We now introduce the concept of E-frames.

Definition 2.15. A sequence $\left\{f_{k}\right\}_{k=1}^{\infty}$ of elements in $\mathcal{H}$ is an E-frame for $\mathcal{H}$ if there exist positive real numbers $A$ and $B$ such that

$$
A\|f\|^{2} \leq\left\|\left\{\left\langle f,\left(E\left\{f_{j}\right\}_{j=1}^{\infty}\right)_{k}\right\rangle\right\}_{k=1}^{\infty}\right\|_{\ell^{2}}^{2} \leq B\|f\|^{2}, \quad \forall f \in \mathcal{H} .
$$

More precisely, the sequence $\left\{f_{k}\right\}_{k=1}^{\infty}$ is an E-frame for $\mathcal{H}$ if its E-transform is a frame for $\mathcal{H}$.

The numbers $A$ and $B$ in (2.7) are called E-frame bounds. They are not unique. The optimal upper E-frame bound is the infimum over all upper E-frame bounds, and the optimal lower frame bound is the supremum over all lower E-frame bounds. Note that the optimal E-bounds actually are E-frame bounds.

A special role is played by E-frames for which the optimal E-frame bounds coincide: 
Definition 2.16. A sequence $\left\{f_{k}\right\}_{k=1}^{\infty}$ of elements in $\mathcal{H}$ is a tight E-frame if there exists constant $A>0$ such that

$$
\left\|\left\{\left\langle f,\left(E\left\{f_{j}\right\}_{j=1}^{\infty}\right)_{k}\right\rangle\right\}_{k=1}^{\infty}\right\|_{\ell^{2}}^{2}=A\|f\|^{2}, \quad \forall f \in \mathcal{H} .
$$

The (exact) number $A$ is called the tight E-frame bound.

It is clear that every E-frame is E-complete. Also, since an E-frame $\left\{f_{k}\right\}_{k=1}^{\infty}$ is an E-Bessel sequence, the operator

$$
\mathrm{T}: \ell^{2} \rightarrow \mathcal{H}, \quad \mathrm{T}\left\{c_{k}\right\}_{k=1}^{\infty}=\sum_{k=1}^{\infty} c_{k}\left(E\left\{f_{j}\right\}_{j=1}^{\infty}\right)_{k},
$$

is bounded. $\mathrm{T}$ is called the pre $\mathrm{E}$-frame operator. Its adjoint, the analysis operator, is given by

$$
\mathrm{T}^{*}: \mathcal{H} \rightarrow \ell^{2}, \mathrm{~T}^{*} f=\left\{\left\langle f,\left(E\left\{f_{j}\right\}_{j=1}^{\infty}\right)_{k}\right\rangle\right\}_{k=1}^{\infty} .
$$

Composing $\mathrm{T}$ and $\mathrm{T}^{*}$, the $\mathrm{E}$-frame operator

$$
\mathrm{S}=\mathrm{TT}^{*}: \mathcal{H} \rightarrow \mathcal{H}, \quad \mathrm{S} f=\sum_{k=1}^{\infty}\left\langle f,\left(E\left\{f_{j}\right\}_{j=1}^{\infty}\right)_{k}\right\rangle\left(E\left\{f_{j}\right\}_{j=1}^{\infty}\right)_{k} .
$$

is obtained. Since $\left\{f_{k}\right\}_{k=1}^{\infty}$ is an E-Bessel sequence, the series defining S converges unconditionally for all $f \in \mathcal{H}$.

The reader can easily check that the E-frame operator $\mathrm{S}$ is bounded, invertible, self-adjoint, and positive; that if $\left\{f_{k}\right\}_{k=1}^{\infty}$ is E-frame with the E-frame bounds $A$ and $B$, then $A \leq \mathrm{S} \leq B$ and $\left\{\mathrm{S}^{-1} f_{k}\right\}_{k=1}^{\infty}$ is an E-frame with E-frame operator $\mathrm{S}^{-1}$ and $\mathrm{E}$-frame bounds $B^{-1}$ and $A^{-1}$; and that if $A$ and $B$ are the optimal E-frame bounds for $\left\{f_{k}\right\}_{k=1}^{\infty}$ then the bounds $B^{-1}$ and $A^{-1}$ are optimal for $\left\{\mathrm{S}^{-1} f_{k}\right\}_{k=1}^{\infty}$.

Moreover, we have the following E-frame decomposition relation which shows that if $\left\{f_{k}\right\}_{k=1}^{\infty}$ is an $\mathrm{E}$-frame for $\mathcal{H}$, then every element in $\mathcal{H}$ has a representation as an infinite linear combination of the $\mathrm{E}$-frame elements. Therefore it is natural to view an E-frame as some kind of generalized E-basis.

Proposition 2.17. Let $\left\{f_{k}\right\}_{k=1}^{\infty}$ be $\mathrm{E}$-frame with the $\mathrm{E}$-frame operator $\mathrm{S}$. Then

$$
f=\sum_{k=1}^{\infty}\left\langle f,\left(E\left\{S^{-1} f_{j}\right\}_{j=1}^{\infty}\right)_{k}\right\rangle\left(E\left\{f_{j}\right\}_{j=1}^{\infty}\right)_{k}, \quad \forall f \in \mathcal{H}
$$

and

$$
f=\sum_{k=1}^{\infty}\left\langle f,\left(E\left\{f_{j}\right\}_{j=1}^{\infty}\right)_{k}\right\rangle\left(E\left\{S^{-1} f_{j}\right\}_{j=1}^{\infty}\right)_{k} . \forall f \in \mathcal{H}
$$

Both series converge unconditionally for all $f \in \mathcal{H}$.

Definition 2.18. Let $\left\{f_{k}\right\}_{k=1}^{\infty}$ be an E-frame for $\mathcal{H}$. An E-frame $\left\{g_{k}\right\}_{k=1}^{\infty}$ is called a dual E-frame of $\left\{f_{k}\right\}_{k=1}^{\infty}$ for $\mathcal{H}$, if

$$
f=\sum_{k=1}^{\infty}\left\langle f,\left(E\left\{g_{j}\right\}_{j=1}^{\infty}\right)_{k}\right\rangle\left(E\left\{f_{j}\right\}_{j=1}^{\infty}\right)_{k}, \quad \forall f \in \mathcal{H}
$$


If $\left\{g_{k}\right\}_{k=1}^{\infty}$ is a dual E-frame of $\left\{f_{k}\right\}_{k=1}^{\infty}$, then $\left\{f_{k}\right\}_{k=1}^{\infty}$ is also a dual E-frame of $\left\{g_{k}\right\}_{k=1}^{\infty}$. For this reason, we will usually call $\left\{f_{k}\right\}_{k=1}^{\infty}$ and $\left\{g_{k}\right\}_{k=1}^{\infty}$ a dual E-frame pair when (2.8) holds. The E-frame $\left\{S^{-1} f_{k}\right\}_{k=1}^{\infty}$ is called the canonical dual E-frame of $\left\{f_{k}\right\}_{k=1}^{\infty}$. It is important to notice that $\left\{\mathrm{S}^{-1} f_{k}\right\}_{k=1}^{\infty}$ plays the same role in E-frame theory as the dual E-Reisz basis theory.

Definition 2.19. Let $\left\{f_{k}\right\}_{k=1}^{\infty}$ be a sequence in $\mathcal{H}$. We say that $\left\{f_{k}\right\}_{k=1}^{\infty}$ is an E-frame sequence if it is an E-frame for $\overline{\operatorname{span}}\left\{\left(E\left\{f_{j}\right\}_{j=1}^{\infty}\right)_{k}\right\}_{k=1}^{\infty}$.

Corollary 2.20. If $\left\{f_{k}\right\}_{k=1}^{\infty}$ is a tight $\mathrm{E}$-frame with the tight $\mathrm{E}$-frame bound $A$, then the canonical dual $\mathrm{E}$-frame is $\left\{A^{-1} f_{k}\right\}_{k=1}^{\infty}$ and

$$
f=\frac{1}{A} \sum_{k=1}^{\infty}\left\langle f,\left(E\left\{f_{j}\right\}_{j=1}^{\infty}\right)_{k}\right\rangle\left(E\left\{f_{j}\right\}_{j=1}^{\infty}\right)_{k} . \quad \forall f \in \mathcal{H}
$$

The following corollary which is a consequence of Proposition 2.10 and uniqueness part of Theorem 2.7, shows that all E-Riesz bases are E-frames.

Corollary 2.21. An E-Riesz basis $\left\{f_{k}\right\}_{k=1}^{\infty}$ for $\mathcal{H}$ is an E-frame for $\mathcal{H}$, and the $\mathrm{E}-$ Riesz basis bounds coincide with the $\mathrm{E}-$ frame bounds. The dual $\mathrm{E}-$ Riesz basis equals the canonical dual $\mathrm{E}$-frame $\left\{\mathrm{S}^{-1} f_{k}\right\}_{k=1}^{\infty}$.

An E-frame that is not E-Riesz basis is said to be overcomplete. As same as in ordinary Riesz basis, an E-frame $\left\{f_{k}\right\}_{k=1}^{\infty}$ is an E-Riesz basis for $\mathcal{H}$ if, and only if, whenever $\sum_{k=1}^{\infty} c_{k}\left(E\left\{f_{j}\right\}_{j=1}^{\infty}\right)_{k}=0$ for some $\left\{c_{k}\right\}_{k=1}^{\infty} \in \ell^{2}$, we have $c_{k}=0$ for all $k \in N$. From this we can easily conclude that every overcomplete E-frame $\left\{f_{k}\right\}_{k=1}^{\infty}$ has other dual E-frames than the canonical; i.e. there exist E-frames $\left\{g_{k}\right\}_{k=1}^{\infty} \neq\left\{S^{-1} f_{k}\right\}_{k=1}^{\infty}$ for which

$$
f=\sum_{k=1}^{\infty}\left\langle f,\left(E\left\{g_{j}\right\}_{j=1}^{\infty}\right)_{k}\right\rangle\left(E\left\{f_{j}\right\}_{j=1}^{\infty}\right)_{k}, \quad \forall f \in \mathcal{H} .
$$

Furthermore, we deduce that for an overcomplete E-frame $\left\{f_{k}\right\}_{k=1}^{\infty}$, there exist several sets of coefficients $\left\{c_{k}\right\}_{k=1}^{\infty} \in \ell^{2}$ for which $f=\sum_{k=1}^{\infty} c_{k}\left(E\left\{f_{j}\right\}_{j=1}^{\infty}\right)$. It is an easy verification to see that the E-frame coefficients $\left\{\left\langle f,\left(E\left\{S^{-1} f_{j}\right\}_{j=1}^{\infty}\right)_{k}\right\rangle\right\}_{k=1}^{\infty}$ have minimal $\ell^{2}$-norm among all sequences representing $f$ which implies easily that for an E-frame $\left\{f_{k}\right\}_{k=1}^{\infty}$ with pre E-frame $\mathrm{T}$ and E-frame operator $\mathrm{S}$, the pseudo-inverse of $\mathrm{T}$ be defined by

$$
\mathrm{T}^{\dagger}: \mathcal{H} \rightarrow \ell^{2}, \quad \mathrm{~T}^{\dagger} f=\left\{\left\langle f,\left(E\left\{S^{-1} f_{j}\right\}_{j=1}^{\infty}\right)_{k}\right\rangle\right\}_{k=1}^{\infty}
$$

The optimal E-frame bounds can be expressed in terms of the operators $\mathrm{T}$ and $\mathrm{S}$ and their inverses and pseudo-inverses. In fact the optimal E-frame bounds $A$ and $B$ for an E-frame $\left\{f_{k}\right\}_{k=1}^{\infty}$ are $A=\left\|\mathrm{S}^{-1}\right\|^{-1}=\left\|\mathrm{T}^{\dagger}\right\|^{-2}$ and $B=\|\mathrm{S}\|=\|\mathrm{T}\|^{2}$.

We know that if $\left\{f_{k}\right\}_{k=1}^{\infty}$ is an E-frame, then $\left\{S^{-1} f_{k}\right\}_{k=1}^{\infty}$ is also an E-frame. In the following a much more general results are stated. We begin with the 
following proposition which can be easily proved by a similar manner as in ([11], Proposition 5.3.1).

Proposition 2.22. Let $\left\{f_{k}\right\}_{k=1}^{\infty}$ be an $\mathrm{E}$-frame for $\mathcal{H}$ with $\mathrm{E}$-frame bounds $A, B$ and let $\mathrm{U}: \mathcal{H} \rightarrow \mathcal{H}$ be a bounded operator with closed range. Then $\left\{U f_{k}\right\}_{k=1}^{\infty}$ is an $\mathrm{E}$-frame sequence with $\mathrm{E}$-frame bounds $A\left\|\mathrm{U}^{\dagger}\right\|^{-2}$ and $B\|\mathrm{U}\|^{2}$.

Corollary 2.23. Suppose that $\left\{f_{k}\right\}_{k=1}^{\infty}$ is an E-frame for $\mathcal{H}$ with E-frame bounds $A, B$ and that $U$ is a bounded surjective operator on $\mathcal{H}$. Then $\left\{U f_{k}\right\}_{k=1}^{\infty}$ is an $\mathrm{E}$-frame with $\mathrm{E}$-frame bounds $A\left\|\mathrm{U}^{\dagger}\right\|^{-2}$ and $B\|\mathrm{U}\|^{2}$.

Corollary 2.24. If $\left\{f_{k}\right\}_{k=1}^{\infty}$ is an $\mathrm{E}$-frame sequence for $\mathcal{H}$ with $\mathrm{E}$-frame bounds $A, B$ and $\mathrm{U}$ is a unitary operator on $\mathcal{H}$, then $\left\{U f_{k}\right\}_{k=1}^{\infty}$ is an $\mathrm{E}$-frame sequence with $\mathrm{E}$-frame bounds $A$ and $B$.

As same as for the ordinary frames, any E-frame can be characterized in terms of the pre E-frame operator. This characterization does not involve any knowledge of the E-frame bounds. Proof of the following proposition can be easily adapted to one of ([11], Theorem 5.4.1).

Proposition 2.25. A sequence $\left\{f_{k}\right\}_{k=1}^{\infty}$ in $\mathcal{H}$ is an $\mathrm{E}$-frame for $\mathcal{H}$ if and only if

$$
\mathrm{T}:\left\{c_{k}\right\}_{k=1}^{\infty} \rightarrow \sum_{k=1}^{\infty} c_{k}\left(E\left\{f_{j}\right\}_{j=1}^{\infty}\right)_{k}
$$

is a well-defined mapping of $\ell^{2}$ onto $\mathcal{H}$.

Corollary 2.26. A sequence $\left\{f_{k}\right\}_{k=1}^{\infty}$ in $\mathcal{H}$ is an E-frame sequence if and only if the pre $\mathrm{E}$-frame operator is well-defined on $\ell^{2}$ and has closed range.

Recall that all E-Riesz bases for the Hilbert space $\mathcal{H}$ are characterized as the families $\left\{\mathrm{U}\left(E^{-1}\left\{e_{i}\right\}_{i=1}^{\infty}\right)_{k}\right\}_{k=1}^{\infty}$, where $\left\{e_{k}\right\}_{k=1}^{\infty}$ is an orthonormal basis for $\mathcal{H}$ and $\mathrm{U}$ is a bounded invertible operator on $\mathcal{H}$. We can now give a similar characterization for E-frames:

Theorem 2.27. Let $\left\{e_{k}\right\}_{k=1}^{\infty}$ be an arbitrary orthonormal basis for $\mathcal{H}$. The E-frames for $\mathcal{H}$ are precisely the families $\left\{\mathrm{U}\left(E^{-1}\left\{e_{i}\right\}_{i=1}^{\infty}\right)_{k}\right\}_{k=1}^{\infty}$, where $\mathrm{U}$ is a bounded surjective operator on $\mathcal{H}$.

Proof. Let $\left\{\delta_{k}\right\}_{k=1}^{\infty}$ be the canonical basis for $\ell^{2}$ and let $\Phi: \mathcal{H} \rightarrow \ell^{2}$ be the isometric isomorphism defined by $\Phi e_{k}=\delta_{k}$. If $\left\{f_{k}\right\}_{k=1}^{\infty}$ is an E-frame, then the pre $\mathrm{E}$-frame operator $\mathrm{T}$ is bounded and surjective, and $\mathrm{T} \delta_{k}=\left(E\left\{f_{j}\right\}_{j=1}^{\infty}\right)_{k}$ Chosen $\mathrm{U}=\mathrm{T} \Phi$, we have $\left\{f_{k}\right\}_{k=1}^{\infty}=\left\{\mathrm{U}\left(E^{-1}\left\{e_{i}\right\}_{i=1}^{\infty}\right)_{k}\right\}_{k=1}^{\infty}$, and $\mathrm{U}$ is bounded and surjective. That every family of the form $\left\{f_{k}\right\}_{k=1}^{\infty}=\left\{\mathrm{U}\left(E^{-1}\left\{e_{i}\right\}_{i=1}^{\infty}\right)_{k}\right\}_{k=1}^{\infty}$, is an $\mathrm{E}$-frame follows from the equality

$$
\begin{aligned}
\sum_{k=1}^{\infty}\left|\left\langle f,\left(E\left\{U\left(E^{-1}\left\{e_{i}\right\}_{i=1}^{\infty}\right)_{j}\right\}_{j=1}^{\infty}\right)_{k}\right\rangle\right|^{2} & =\sum_{k=1}^{\infty}\left|\left\langle f, U\left(E\left\{\left(E^{-1}\left\{e_{i}\right\}_{i=1}^{\infty}\right)_{j}\right\}_{j=1}^{\infty}\right){ }_{k}\right\rangle\right|^{2} \\
& =\sum_{k=1}^{\infty}\left|\left\langle f, U e_{k}\right\rangle\right|^{2}=\left\|U^{*} f\right\|^{2},
\end{aligned}
$$


together with ([11], Lemma 2.4.1). This completes the proof.

In the rest of this paper we are going to characterize all dual E-frames $\left\{g_{k}\right\}_{k=1}^{\infty}$ associated with a given E-frame $\left\{f_{k}\right\}_{k=1}^{\infty}$. We recall that two E-frames $\left\{f_{k}\right\}_{k=1}^{\infty}$ and $\left\{g_{k}\right\}_{k=1}^{\infty}$ are dual E-frames if for all $f \in \mathcal{H}$ we have

$$
f=\sum_{k=1}^{\infty}\left\langle f,\left(E\left\{f_{j}\right\}_{j=1}^{\infty}\right)_{k}\right\rangle\left(E\left\{g_{j}\right\}_{j=1}^{\infty}\right)_{k}=\sum_{k=1}^{\infty}\left\langle f,\left(E\left\{g_{j}\right\}_{j=1}^{\infty}\right)_{k}\right\rangle\left(E\left\{f_{j}\right\}_{j=1}^{\infty}\right)_{k} .
$$

Theorem 2.28. Let $\left\{f_{k}\right\}_{k=1}^{\infty}$ be an E-frame for $\mathcal{H}$. The dual E-frames of $\left\{f_{k}\right\}_{k=1}^{\infty}$ are precisely the families

$$
\left\{g_{k}\right\}_{k=1}^{\infty}=\left\{\mathrm{S}^{-1} f_{k}+h_{k}-\sum_{n=1}^{\infty}\left\langle\left(E\left\{\mathrm{~S}^{-1} f_{j}\right\}_{j=1}^{\infty}\right)_{k},\left(E\left\{f_{j}\right\}_{j=1}^{\infty}\right)_{n}\right\rangle h_{n}\right\}_{k=1}^{\infty}
$$

where $\left\{h_{k}\right\}_{k=1}^{\infty}$ is an $\mathrm{E}-$ Bessel sequence in $\mathcal{H}$.

Proof. We first prove that the sequence $\left\{g_{k}\right\}_{k=1}^{\infty}$ given by (2.9) is an E-Bessel sequence in $\mathcal{H}$. Let $A$ and $B$ be the lower and upper E-frame bounds for $\left\{f_{k}\right\}_{k=1}^{\infty}$ and $\left\{h_{k}\right\}_{k=1}^{\infty}$ be an E-Bessel sequence in $\mathcal{H}$ with the E-Bessel bound $C$. For $f \in \mathcal{H}$, we have

$$
\begin{aligned}
& \sum_{k=1}^{\infty}\left|\left\langle f,\left(E\left\{g_{j}\right\}_{j=1}^{\infty}\right)_{k}\right\rangle\right|^{2} \\
= & \sum_{k}^{\infty}\left|\left\langle f,\left(E\left\{S^{-1} f_{j}\right\}_{j=1}^{\infty}\right)_{k}+\left(E\left\{h_{j}\right\}_{j=1}^{\infty}\right)_{k}-\sum_{n=1}^{\infty}\left\langle\left(E\left\{S^{-1} f_{j}\right\}_{j=1}^{\infty}\right)_{k},\left(E\left\{f_{j}\right\}_{j=1}^{\infty}\right)_{n}\right\rangle\left(E\left\{h_{j}\right\}_{j=1}^{\infty}\right)_{n}\right\rangle\right|^{2} \\
= & \sum_{k}^{\infty} \mid\left\langle f,\left(E\left\{S^{-1} f_{j}\right\}_{j=1}^{\infty}\right)_{k}\right\rangle+\left\langle f,\left(E\left\{h_{j}\right\}_{j=1}^{\infty}\right)_{k}\right\rangle \\
& -\left.\sum_{n=1}^{\infty}\left\langle\left(E\left\{f_{j}\right\}_{j=1}^{\infty}\right)_{n},\left(E\left\{S^{-1} f_{j}\right\}_{j=1}^{\infty}\right)_{k}\right\rangle\left\langle f,\left(E\left\{h_{j}\right\}_{j=1}^{\infty}\right)_{n}\right\rangle\right|^{2} \\
\leq & \sum_{k}^{\infty}\left\{\left|\left\langle f,\left(E\left\{S^{-1} f_{j}\right\}_{j=1}^{\infty}\right)_{k}\right\rangle\right|+\left|\left\langle f,\left(E\left\{h_{j}\right\}_{j=1}^{\infty}\right)_{k}\right\rangle\right|\right. \\
& \left.+\sum_{n=1}^{\infty}\left|\left\langle\left(E\left\{f_{j}\right\}_{j=1}^{\infty}\right)_{n},\left(E\left\{S^{-1} f_{j}\right\}_{j=1}^{\infty}\right)_{k}\right\rangle\left\langle f,\left(E\left\{h_{j}\right\}_{j=1}^{\infty}\right)_{n}\right\rangle\right|\right\}^{2}
\end{aligned}
$$




$$
\begin{aligned}
& \stackrel{\text { Hölder }}{\leq} \sum_{k}^{\infty}\left\{\left|\left\langle f,\left(E\left\{S^{-1} f_{j}\right\}_{j=1}^{\infty}\right)_{k}\right\rangle\right|+\left|\left\langle f,\left(E\left\{h_{j}\right\}_{j=1}^{\infty}\right)_{k}\right\rangle\right|\right. \\
& \left.+\left(\sum_{n=1}^{\infty}\left|\left\langle\left(E\left\{f_{j}\right\}_{j=1}^{\infty}\right)_{n},\left(E\left\{S^{-1} f_{j}\right\}_{j=1}^{\infty}\right)_{k}\right\rangle\right|^{2}\right)^{1 / 2}\left(\sum_{n=1}^{\infty}\left|\left\langle f,\left(E\left\{h_{j}\right\}_{j=1}^{\infty}\right)_{n}\right\rangle\right|^{2}\right)^{1 / 2}\right\}^{2} \\
& \leq \sum_{k}^{\infty}\left\{\left|\left\langle f,\left(E\left\{S^{-1} f_{j}\right\}_{j=1}^{\infty}\right)_{k}\right\rangle\right|+\left|\left\langle f,\left(E\left\{h_{j}\right\}_{j=1}^{\infty}\right)_{k}\right\rangle\right|+B^{1 / 2}\left\|\left(E\left\{S^{-1} f_{j}\right\}_{j=1}^{\infty}\right)_{k}\right\| C^{1 / 2}\|f\|\right\}^{2} \\
& \leq \sum_{k}^{\infty} 2\left(\left\{\left|\left\langle f,\left(E\left\{S^{-1} f_{j}\right\}_{j=1}^{\infty}\right)_{k}\right\rangle\right|+\left|\left\langle f,\left(E\left\{h_{j}\right\}_{j=1}^{\infty}\right)_{k}\right\rangle\right|\right\}^{2}+\left\{B^{1 / 2}\left\|\left(E\left\{S^{-1} f_{j}\right\}_{j=1}^{\infty}\right)_{k}\right\| C^{1 / 2}\|f\|\right\}^{2}\right) \\
& \leq 2 \sum_{k}^{\infty}\left\{\left|\left\langle f,\left(E\left\{S^{-1} f_{j}\right\}_{j=1}^{\infty}\right)_{k}\right\rangle\right|+\left|\left\langle f,\left(E\left\{h_{j}\right\}_{j=1}^{\infty}\right)_{k}\right\rangle\right|\right\}^{2}+2 B C\|f\|^{2} \underbrace{\sum_{k}^{\infty}\left\|\left(E\left\{S^{-1} f_{j}\right\}_{j=1}^{\infty}\right)_{k}\right\|^{2}}_{:=M<\infty} \\
& \leq 2 \sum_{k}^{\infty}\left\{\left|\left\langle f,\left(E\left\{S^{-1} f_{j}\right\}_{j=1}^{\infty}\right)_{k}\right\rangle\right|+\left|\left\langle f,\left(E\left\{h_{j}\right\}_{j=1}^{\infty}\right)_{k}\right\rangle\right|\right\}^{2}+2 B C M\|f\|^{2} \\
& \leq 4 \sum_{k}^{\infty}\left|\left\langle f,\left(E\left\{S^{-1} f_{j}\right\}_{j=1}^{\infty}\right)_{k}\right\rangle\right|^{2}+4 \sum_{k}^{\infty}\left|\left\langle f,\left(E\left\{h_{j}\right\}_{j=1}^{\infty}\right)_{k}\right\rangle\right|^{2}+2 B C M\|f\|^{2} \\
& \leq 4 A^{-1}\|f\|^{2}+4 C\|f\|^{2}+2 B C M\|f\|^{2} \\
& \leq\left(4 A^{-1}+4 C+2 B C M\right)\|f\|^{2},
\end{aligned}
$$

which implies that $\left\{g_{k}\right\}_{k=1}^{\infty}$ is an E-Bessel sequence with the E-Bessel bound $\left(4 A^{-1}+4 C+2 B C M\right)$. For the rest of the proof, let $f \in \mathcal{H}$. We have

$$
\begin{aligned}
& \sum_{k=1}^{\infty}\left\langle f,\left(E\left\{g_{j}\right\}_{j=1}^{\infty}\right)_{k}\right\rangle\left(E\left\{f_{j}\right\}_{j=1}^{\infty}\right)_{k} \\
= & \sum_{k=1}^{\infty}\left\langle f,\left(E\left\{\mathrm{~S}^{-1} f_{j}\right\}_{j=1}^{\infty}\right)_{k}+\left(E\left\{h_{j}\right\}_{j=1}^{\infty}\right)_{k}-\sum_{n=1}^{\infty}\left\langle\left(E\left\{\mathrm{~S}^{-1} f_{j}\right\}_{j=1}^{\infty}\right)_{k},\left(E\left\{f_{j}\right\}_{j=1}^{\infty}\right)_{n}\right\rangle\left(E\left\{h_{j}\right\}_{j=1}^{\infty}\right)_{k}\right\rangle
\end{aligned}
$$


In the same way, we have

$$
\sum_{k=1}^{\infty}\left\langle f,\left(E\left\{f_{j}\right\}_{j=1}^{\infty}\right)\right\rangle_{k}\left(E\left\{g_{j}\right\}_{j=1}^{\infty}\right)_{k}=f, \quad \forall f \in \mathcal{H} .
$$

Therefore, $\left\{f_{k}\right\}_{k=1}^{\infty}$ and $\left\{g_{k}\right\}_{k=1}^{\infty}$ are dual E-frame of each other and the proof is now completed.

Note that if $\left\{f_{k}\right\}_{k=1}^{\infty}$ be an E-Riesz basis, then $\left\{f_{k}\right\}_{k=1}^{\infty}$ and $\left\{\mathrm{S}^{-1} f_{k}\right\}_{k=1}^{\infty}$ are E-biorthogonal by Corollary 2.21. Thus, independently of the choice of $\left\{h_{k}\right\}_{k=1}^{\infty}$, the element $g_{k}$ in (2.9) is given by

$$
\begin{aligned}
g_{k} & =\mathrm{S}^{-1} f_{k}+h_{k}-\sum_{n=1}^{\infty}\left\langle\left(E\left\{\mathrm{~S}^{-1} f_{j}\right\}_{j=1}^{\infty}\right)_{k},\left(E\left\{f_{j}\right\}_{j=1}^{\infty}\right)_{n}\right\rangle h_{n} \\
& =\mathrm{S}^{-1} f_{k}+h_{k}-h_{k} \\
& =\mathrm{S}^{-1} f_{k} .
\end{aligned}
$$

This shows that an E-Riesz basis $\left\{f_{k}\right\}_{k=1}^{\infty}$ has a unique dual E-frame, as state before in Theorem 2.7.

\section{Special CASES}

In this section we are going to introduce some special cases of E-frames.

3.1. I-frames. Let I be the identity operator on $\underset{n=1}{\infty} \mathcal{H}$. We observe that the I-frames are reduced to the ordinary frames and deduce that the E-frames are actually generalized the concept of frames for separable Hilbert space $\mathcal{H}$. Applying the characterization results in the previous section, we conclude that the orthonormal bases for $\mathcal{H}$ are precisely the sets $\left\{\mathrm{U} e_{k}\right\}_{k=1}^{\infty}$, where $\mathrm{U}$ is an unitary operator on $\mathcal{H}$; the Riesz bases are the families of the form $\left\{\mathrm{U} e_{k}\right\}_{k=1}^{\infty}$, where $\mathrm{U}$ is a bounded bijective operator on $\mathcal{H}$ and the frames are precisely the families $\left\{\mathrm{U} e_{k}\right\}_{k=1}^{\infty}$, where $\mathrm{U}$ is bounded surjective operator on $\mathcal{H}$. Also, if $\left\{f_{k}\right\}_{k=1}^{\infty}$ be a frame for $\mathcal{H}$, then the dual frames of $\left\{f_{k}\right\}_{k=1}^{\infty}$ are precisely the families

$$
\left\{g_{k}\right\}_{k=1}^{\infty}=\left\{\mathrm{S}^{-1} f_{k}+h_{k}-\sum_{j=1}^{\infty}\left\langle\mathrm{S}^{-1} f_{k}, f_{j}\right\rangle h_{j}\right\}_{k=1}^{\infty}
$$

where $\left\{h_{k}\right\}_{k=1}^{\infty}$ is a Bessel sequence in $\mathcal{H}$.

3.2. $\Delta$-frames and bounded below operators on $b v_{p}$. For $0<p<\infty$, the $p$-bounded variation sequence space $b v_{p}$ is defined as the space of all real or complex sequences $x=\left\{x_{k}\right\}_{k=1}^{\infty}$ for which

$$
\|x\|_{b v_{p}}:=\left(\sum_{k=1}^{\infty}\left|x_{k}-x_{k-1}\right|^{p}\right)^{\frac{1}{p}}<\infty
$$


where $x_{0}=0$. More precisely, the $b v_{p}$ is the space of all real or complex sequences whose $\boldsymbol{\Delta}$-transforms are in the space $\ell^{p}$, where $\boldsymbol{\Delta}$ denotes the matrix $\boldsymbol{\Delta}=$ $\left(\boldsymbol{\Delta}_{n, k}\right)_{n, k \geq 1}$ defined by

$$
\boldsymbol{\Delta}_{n, k}= \begin{cases}(-1)^{n-k} & n-1 \leq k \leq n, \\ 0 & 1 \leq k<n-1 \text { or } k>n,\end{cases}
$$

and the matrix form of $\boldsymbol{\Delta}$ is

$$
\boldsymbol{\Delta}=\left(\begin{array}{cccccc}
1 & 0 & 0 & 0 & 0 & \cdots \\
-1 & 1 & 0 & 0 & 0 & \cdots \\
0 & -1 & 1 & 0 & 0 & \cdots \\
0 & 0 & -1 & 1 & 0 & \cdots \\
0 & 0 & 0 & -1 & 1 & \cdots \\
\vdots & \vdots & \vdots & \vdots & \ddots & \ddots
\end{array}\right)
$$

This space was introduced and studied by Altay and Basąr in [3]. They proved that the sequence space $b v_{p}$ is linearly isomorphic to the space $\ell^{p}$ and that the space $b v_{2}$ is a Hilbert space.

In this section, we first consider the lower bound problem for some matrix mapping on the space $b v_{p}$. The lower bound involved here, is the number $L_{b v_{p}, b v_{p}}(\mathrm{~A})$, which is defined as the supremum of those $l$, obeying the following inequality

$$
\|\mathrm{A} x\|_{b v_{p}} \geq l\|x\|_{b v_{p}},
$$

where $x \geq 0, x \in b v_{p}$ and $\mathrm{A}=\left(a_{n, k}\right)_{n, k \geq 1}$ is a non-negative lower triangular matrix operator selfmap of the space $b v_{p}, 0<p<1$. Our result gives a lower estimate for $L_{b v_{p}, b v_{p}}\left(A^{t}\right)$ in term of the constant $M$, defined by

$$
\left(a_{n, k}-a_{n, k-1}\right) \leq M\left(a_{n, j}-a_{n, j-1}\right), \quad 1 \leq k \leq j \leq n .
$$

We use the convention that any term with zero subscript is equal to naught. Here $M \geq 1$ and we shall assume that $M$ is the smallest value appeared in (3.1). If (3.1) is fails, we set $M=\infty$.

Theorem 3.1. Let $0<p<1$ and $A=\left(a_{n, k}\right)_{n, k \geq 1}$ be a lower triangular matrix with non-negative entries. If $a_{n, k} \leq a_{n, k+1}(1 \leq k<n)$, then

$$
b v_{p}, b v_{p}\left(A^{t}\right) \geq \frac{1}{2} p M^{p-1}\left(\inf _{j \geq 1} a_{j, j}\right) .
$$

Here $M$ is defined by (3.1).

Proof. Let $x \geq 0$ with $\|x\|_{b v_{p}}=1$. Since $p-1<0$, (3.1) and Lemma 3.13 of [5] with Fubini's theorem follows that

$$
\begin{aligned}
\left\|A^{t} x\right\|_{b v_{p}}^{p} & =\sum_{k=1}^{\infty}\left(\sum_{n=k}^{\infty}\left(a_{n, k}-a_{n, k-1}\right) x_{n}\right)^{p} \\
& \geq p\left(\sum_{k=1}^{\infty} \sum_{j=k}^{\infty}\left(a_{j, k}-a_{j, k-1}\right) x_{j}\left(\sum_{n=j}^{\infty}\left(a_{n, k}-a_{n, k-1}\right) x_{n}\right)^{p-1}\right) \\
& \geq p M^{p-1} \sum_{k=1}^{\infty} \sum_{j=k}^{\infty}\left(a_{j, k}-a_{j, k-1}\right) x_{j}\left(\sum_{n=j}^{\infty}\left(a_{n, j}-a_{n, j-1}\right) x_{n}\right)^{p-1}
\end{aligned}
$$




$$
=p M^{p-1} \sum_{j=1}^{\infty} a_{j, j} x_{j}\left(\sum_{n=j}^{\infty}\left(a_{n, j}-a_{n, j-1}\right) x_{n}\right)^{p-1},
$$

where $a_{n, 0}=0$ for all $n \geq 1$. Applying Hölder's inequality with the inequality $\|x\|_{b v_{p}} \leq 2\|x\|_{p}$, we have

$$
\begin{aligned}
\sum_{j=1}^{\infty} a_{j, j} x_{j}\left(\sum_{n=j}^{\infty}\left(a_{n, j}-a_{n, j-1}\right) x_{n}\right)^{p-1} & \\
& \geq\left(\sum_{j=1}^{\infty}\left(a_{j, j} x_{j}\right)^{p}\right)^{\frac{1}{p}}\left(\sum_{j=1}^{\infty}\left(\sum_{n=j}^{\infty}\left(a_{n, j}-a_{n, j-1}\right) x_{n}\right)^{p}\right)^{\frac{p-1}{p}} \\
& \geq\left(\inf _{j \geq 1} a_{j, j}\right)\left\|A^{t} x\right\|_{b v_{p}}^{p-1}\|x\|_{p} \\
& \geq \frac{1}{2}\left(\inf _{j \geq 1} a_{j, j}\right)\left\|A^{t} x\right\|_{b v_{p}}^{p-1}\|x\|_{b v_{p}} .
\end{aligned}
$$

Inserting this estimate into the corresponding term in (3.3) gives $\left\|A^{t} x\right\|_{b v_{p}} \geq$ $\frac{1}{2} p M^{p-1}\left(\inf _{j \geq 1} a_{j, j}\right)\|x\|_{b v_{p}}$. This leads us to the lower estimate in (3.2) and completes the proof.

In the following corollaries we apply Theorem 3.1 to some famous classes of non-negative lower triangular matrices such as weighted mean matrices and Nörlund matrices, where the weighted mean matrices, $\left(A_{W}^{W M}\right)=\left(a_{n, k}\right)_{n, k \geq 1}$ and the Nörlund matrices, $\left(A_{W}^{N M}\right)=\left(b_{n, k}\right)_{n, k \geq 1}$, are defined by

$$
a_{n, k}=\left\{\begin{array}{cl}
\frac{w^{\prime}{ }_{n}}{W^{\prime}{ }_{n}} & 1 \leq k \leq n, \\
0 & \text { otherwise, }
\end{array} \quad \& \quad b_{n, k}=\left\{\begin{array}{cl}
\frac{w^{\prime}{ }_{n-k+1}}{W^{\prime}{ }_{n}} & 1 \leq k \leq n, \\
0 & \text { otherwise }
\end{array}\right.\right.
$$

Here $W_{n}^{\prime}=\sum_{k=1}^{n} w_{k}^{\prime}$ and $w^{\prime}=\left(w_{n}^{\prime}\right)$ is a non negative sequence with $w_{1}^{\prime}>0$.

Corollary 3.2. Let $0<p<1$ and $w^{\prime}=\left(w_{n}^{\prime}\right)$ be an increasing non-negative sequence of real numbers. Then

$$
L_{b v_{p}, b v_{p}}\left(\left(A_{W}^{W M}\right)^{t}\right) \geq \frac{1}{2} p M^{p-1}\left(\inf _{n \geq 1} \frac{w_{n}^{\prime}}{w_{1}^{\prime}+\ldots+w_{n}^{\prime}}\right),
$$

where $M$ is defined by (3.1).

Corollary 3.2 provides an analogue to those given in ([9], Corollary 4.2) and ([21], Corollary 2.7).

Corollary 3.3. Let $0<p<1$ and $w^{\prime}=\left(w_{n}^{\prime}\right)$ be a decreasing non-negative sequence of real numbers with $w_{1}^{\prime}>0$. Then

$$
L_{b v_{p}, b v_{p}}\left(\left(A_{W}^{N M}\right)^{t}\right) \geq \frac{1}{2} p M^{p-1}\left(\inf _{n \geq 1} \frac{w_{1}^{\prime}}{w_{1}^{\prime}+\ldots+w_{n}^{\prime}}\right),
$$

where $M$ is defined by (3.1). 
Corollary 3.3 provides an analogue to those given in ([9], Corollary 4.3) and ([21], Corollary 2.8).

By definition a $\boldsymbol{\Delta}$-frame for $\mathcal{H}$ is a sequence $\left\{f_{k}\right\}_{k=1}^{\infty}$ for which there exist positive real numbers $A$ and $B$ such that

$$
A\|f\|^{2} \leq\left\|\left\{\left\langle f, f_{k}-f_{k-1}\right\rangle\right\}_{k=1}^{\infty}\right\|_{\ell^{2}}^{2} \leq B\|f\|^{2}, \quad \forall f \in \mathcal{H} .
$$

Since $\|x\|_{b v_{2}}=\|\Delta x\|_{\ell^{2}}$, the definition of a $\Delta$-frame can be modified as any sequence $\left\{f_{k}\right\}_{k=1}^{\infty}$ for which there exist positive real numbers $A$ and $B$ such that

$$
A\|f\|^{2} \leq\left\|\left\{\left\langle f, f_{k}\right\rangle\right\}_{k=1}^{\infty}\right\|_{b v_{2}}^{2} \leq B\|f\|^{2}, \quad \forall f \in \mathcal{H}
$$

We observe that $\boldsymbol{\Delta}$-frames are related to the space $b v_{2}$.

Theorem 3.4. Let $\left\{f_{k}\right\}_{k=1}^{\infty}$ be a Bessel sequence with Bessel bound B. Then it is a $\boldsymbol{\Delta}$-Bessel sequence with $\boldsymbol{\Delta}$-Bessel bound $4 B$.

Proof. Let $f \in \mathcal{H}$. Since $\left\{f_{k}\right\}_{k=1}^{\infty}$ is a Bessel(I-Bessel) sequence with the Bessel bound $B$, we have

$$
\begin{aligned}
\left\|\left\{\left\langle f, f_{k}\right\rangle\right\}_{k=1}^{\infty}\right\|_{b v_{2}}^{2} & =\sum_{k=1}^{\infty}\left|\left\langle f, f_{k}\right\rangle-\left\langle f, f_{k-1}\right\rangle\right|^{2} \\
& \leq \sum_{k=1}^{\infty} 2\left(\left|\left\langle f, f_{k}\right\rangle\right|^{2}+\left|\left\langle f, f_{k-1}\right\rangle\right|^{2}\right) \\
& =2\left(\sum_{k=1}^{\infty}\left|\left\langle f, f_{k}\right\rangle\right|^{2}+\sum_{k=1}^{\infty}\left|\left\langle f, f_{k-1}\right\rangle\right|^{2}\right) \\
& =4 \sum_{k=1}^{\infty}\left|\left\langle f, f_{k}\right\rangle\right|^{2} \\
& \leq 4 B\|f\|^{2},
\end{aligned}
$$

which implies that $\left\{f_{k}\right\}_{k=1}^{\infty}$ is a $\boldsymbol{\Delta}$-Bessel sequence with $\boldsymbol{\Delta}$-Bessel bound $4 B$.

Proposition 3.5. Let $\left\{f_{k}\right\}_{k=1}^{\infty}$ be a $\boldsymbol{\Delta}$-frame for $\mathcal{H}$ with the lower $\boldsymbol{\Delta}$-frame bound $A$. Then it satisfies the lower frame condition with the lower frame bound A/4.

Proof. The proof is a consequence of the following inequalities

$$
A\|f\|^{2} \leq\left\|\left\{\left\langle f, f_{k}\right\rangle\right\}_{k=1}^{\infty}\right\|_{b v_{2}}^{2} \leq 4\left\|\left\{\left\langle f, f_{k}\right\rangle\right\}_{k=1}^{\infty}\right\|_{\ell^{2}}^{2} .
$$

Theorem 3.6. In a Hilbert space $\mathcal{H}$,

(i) There exists a frame which is not a $\Delta$-frame.

(ii) There exists a $\boldsymbol{\Delta}$-frame which is not a frame.

(iii) There exist sequences which are both frame and $\boldsymbol{\Delta}$-frame. 
Proof. (i) Consider the sequence $\left\{f_{k}\right\}_{k=1}^{\infty}=\left\{e_{1}, e_{1},-e_{1},-e_{2}, e_{3}, e_{3}, \cdots\right\}$, where $\left\{e_{k}\right\}_{k=1}^{\infty}$ is an orthonormal basis for $\mathcal{H}$. Then $\left\{f_{k}\right\}_{k=1}^{\infty}$ is a tight frame with frame bound 2 . On the other hand, for any $f \in \mathcal{H}$, we have

$$
\left\|\left\{\left\langle f, f_{k}\right\rangle\right\}_{k=1}^{\infty}\right\|_{b v_{2}}^{2}=\left|\left\langle f, e_{1}\right\rangle\right|^{2}+\sum_{k=1}^{\infty}\left|\left\langle f, e_{k}+e_{k+1}\right\rangle\right|^{2}
$$

Now by the same reason as in ([11], Example 5.1.10), we conclude that $\left\{f_{k}\right\}_{k=1}^{\infty}$ does not satisfy the lower $\boldsymbol{\Delta}$-frame condition.

(ii) The sequence

$$
\left\{f_{k}\right\}_{k=1}^{\infty}=\left\{e_{1}, e_{1}+e_{2}, e_{1}+e_{2}+e_{3}, \cdots\right\}
$$

is a $\Delta$-frame which is not a frame.

(iii) Let $\left\{f_{k}\right\}_{k=1}^{\infty}$ be an arbitrary frame in $\mathcal{H}$ with the frame bound $A$ and $B$. Then the sequence $\left\{g_{k}\right\}_{k=1}^{\infty}=\left\{f_{1}, 0, f_{2}, 0, f_{3}, 0, \ldots\right\}$ is both a frame for $\mathcal{H}$ with the same frame bounds as $\left\{f_{k}\right\}_{k=1}^{\infty}$ and a $\boldsymbol{\Delta}$-frame for $\mathcal{H}$ with the $\boldsymbol{\Delta}$-frame bounds $A$ and $2 B$.

We have the following characterization for $\boldsymbol{\Delta}$-ortonormal bases, $\boldsymbol{\Delta}$-Riesz bases and $\boldsymbol{\Delta}$-frames:

Corollary 3.7. Let $\left\{e_{k}\right\}_{k=1}^{\infty}$ be an orthonormal basis for $\mathcal{H}$. Then

(i) The $\boldsymbol{\Delta}$-orthonormal bases are precisely the sets $\left\{U\left(e_{1}+e_{2}+\cdots+e_{k}\right)\right\}_{k=1}^{\infty}$, where $U$ is an unitary operator on $\mathcal{H}$.

(ii) $A \boldsymbol{\Delta}$-Riesz basis for $\mathcal{H}$ is a family of the form $\left\{U\left(e_{1}+e_{2}+\ldots+e_{k}\right)\right\}_{k=1}^{\infty}$, where $U$ is a bounded bijective operator on $\mathcal{H}$.

(iii) The $\boldsymbol{\Delta}$-frames for $\mathcal{H}$ are precisely the families $\left\{\mathrm{U}\left(e_{1}+e_{2}+\cdots+e_{k}\right)\right\}_{k=1}^{\infty}$, where $\mathrm{U}$ is bounded and surjective operator on $\mathcal{H}$.

Corollary 3.8. Let $\left\{f_{k}\right\}_{k=1}^{\infty}$ be a $\boldsymbol{\Delta}$-frame for $\mathcal{H}$. The dual $\boldsymbol{\Delta}$-frames of $\left\{f_{k}\right\}_{k=1}^{\infty}$ are precisely the families

$$
\left\{g_{k}\right\}_{k=1}^{\infty}=\left\{\mathrm{S}^{-1} f_{k}+h_{k}-\sum_{j=1}^{\infty}\left\langle\mathrm{S}^{-1} f_{k}-\mathrm{S}^{-1} f_{k-1}, f_{j}-f_{j-1}\right\rangle h_{j}\right\}_{k=1}^{\infty}
$$

where $\left\{h_{k}\right\}_{k=1}^{\infty}$ is a $\boldsymbol{\Delta}$-Bessel sequence in $\mathcal{H}$.

3.3. Hausdorff Frames. Let $d \mu$ be the Borel probability measure on $[0,1]$ and $\mathrm{H}_{\mu}=\mathrm{H}_{\mu}(\alpha)=\left(h_{n, k}(\alpha)\right)_{n, k \geq 1}$ be the Hausdorff matrix associated with $d \mu$, defined by

$$
h_{n, k}(\alpha)=\left\{\begin{array}{cc}
\left(\begin{array}{c}
n-1 \\
k-1
\end{array}\right) \int_{0}^{1} \alpha^{k-1}(1-\alpha)^{n-k} d \mu(\alpha) & 1 \leq k \leq n, \\
0 & k>n,
\end{array}\right.
$$


By definition a Hausdorff frame or a $\mathrm{H}_{\mu}$-frame for $\mathcal{H}$ is a sequence $\left\{f_{k}\right\}_{k=1}^{\infty}$ for which there exist positive real numbers $A$ and $B$ such that

$$
A\|f\|^{2} \leq\left\|\left\{\left\langle f, \sum_{k=1}^{n}\left(\begin{array}{c}
n-1 \\
k-1
\end{array}\right) \int_{0}^{1}(1-\alpha)^{n-k} \alpha^{k-1} d \mu(\alpha) f_{k}\right\rangle\right\}_{n=1}^{\infty}\right\|_{\ell^{2}}^{2} \leq B\|f\|^{2}, \quad \forall f \in \mathcal{H} .
$$

Since $\|x\|_{\mathrm{H}_{\mu}}=\left\|\mathrm{H}_{\mu} x\right\|_{\ell^{2}}$, the definition of a $\mathrm{H}_{\mu}$-frame can be modified as any sequence $\left\{f_{k}\right\}_{k=1}^{\infty}$ for which there exist positive real numbers $A$ and $B$ such that

$$
A\|f\|^{2} \leq\left\|\left\{\left\langle f, f_{k}\right\rangle\right\}_{k=1}^{\infty}\right\|_{\mathrm{H}_{\mu}} \leq B\|f\|^{2}, \quad \forall f \in \mathcal{H}
$$

Theorem 3.9. Let $\left\{f_{k}\right\}_{k=1}^{\infty}$ be a Bessel sequence with the Bessel bound $B$ and let $\int_{0}^{1} \alpha^{-1 / 2} d \mu(\alpha)<\infty$. Then it is a Hausdorff Bessel sequence with the Hausdorff Bessel bound $B\left(\int_{0}^{1} \alpha^{-1 / 2} d \mu(\alpha)\right)$.

Proof. Let $f \in H$. By ([19], Theorem 2.1), we have

$$
\begin{gathered}
\left\|\left\{\left\langle f, \sum_{k=1}^{n}\left(\begin{array}{c}
n-1 \\
k-1
\end{array}\right) \int_{0}^{1}(1-\alpha)^{n-k} \alpha^{k-1} d \mu(\alpha) f_{k}\right\rangle\right\}_{n=1}^{\infty}\right\|_{\ell^{2}}^{2} \\
<\left(\int_{0}^{1} \alpha^{-1 / 2} d \mu(\alpha)\right) \sum_{k=1}^{\infty}\left|\left\langle f, f_{k}\right\rangle\right|^{2} \\
\leq B\left(\int_{0}^{1} \alpha^{-1 / 2} d \mu(\alpha)\right)\|f\|^{2} .
\end{gathered}
$$

which implies that $\left\{f_{k}\right\}_{k=1}^{\infty}$ is a Hausdorff Bessel sequence with the desired Hausdorff Bessel bound.

Proposition 3.10. Let $\left\{f_{k}\right\}_{k=1}^{\infty}$ be a Hausdorff frame for $\mathcal{H}$ with the lower Hausdorff frame bound $A$ and $\int_{0}^{1} \alpha^{-1 / 2} d \mu(\alpha)<\infty$. Then it satisfies the lower frame condition with the lower frame bound $A\left(\int_{0}^{1} \alpha^{-1 / 2} d \mu(\alpha)\right)^{-1}$.

Proof. The proof is a consequence of the following inequalities

$$
\begin{aligned}
A\|f\|^{2} \leq\left\|\left\{\left\langle f, \sum_{k=1}^{n}\left(\begin{array}{c}
n-1 \\
k-1
\end{array}\right) \int_{0}^{1}(1-\alpha)^{n-k} \alpha^{k-1} d \mu(\alpha) f_{k}\right\rangle\right\}_{n=1}^{\infty}\right\|_{\ell^{2}}^{2} \\
\leq\left(\int_{0}^{1} \alpha^{-1 / 2} d \mu(\alpha)\right)\left\|\left\{\left\langle f, f_{k}\right\rangle\right\}_{k=1}^{\infty}\right\|_{\ell^{2}}^{2} .
\end{aligned}
$$

We have the following characterization for Hausdorff orthonormal bases, Hausdorff Riesz bases and Hausdorff frames:

Corollary 3.11. Let $\left\{e_{k}\right\}_{k=1}^{\infty}$ be an orthonormal basis for $\mathcal{H}$ and suppose that $\mu_{k}=\int_{0}^{1} \alpha^{k-1} d \mu(\alpha) \neq 0$, for all $k \in N$ and let $\triangle$ be the forward difference operator defined by $\triangle \mu_{k}=\mu_{k}-\mu_{k+1}$ and $\triangle^{n+1} \mu_{k}=\triangle\left(\triangle^{n} \mu_{k}\right)$. Then 
(i) The Hausdorff orthonormal bases are precisely the families

$$
\left\{U\left(\sum_{k=1}^{n}\left(\begin{array}{c}
n-1 \\
k-1
\end{array}\right) \triangle^{n-k} \mu_{k}^{-1} e_{k}\right)\right\}_{n=1}^{\infty}
$$

where $U$ is an unitary operator on $\mathcal{H}$.

(ii) A Hausdorff Riesz basis for $\mathcal{H}$ is a family of the form

$$
\left\{U\left(\sum_{k=1}^{n}\left(\begin{array}{c}
n-1 \\
k-1
\end{array}\right) \triangle^{n-k} \mu_{k}^{-1} e_{k}\right)\right\}_{n=1}^{\infty}
$$

where $U$ is a bounded bijective operator on $\mathcal{H}$.

(iii) The Hausdorff frames for $\mathcal{H}$ are precisely the families

$$
\left\{U\left(\sum_{k=1}^{n}\left(\begin{array}{c}
n-1 \\
k-1
\end{array}\right) \Delta^{n-k} \mu_{k}^{-1} e_{k}\right)\right\}_{n=1}^{\infty}
$$

where $\mathrm{U}$ is bounded and surjective operator on $\mathcal{H}$.

Corollary 3.12. Let $\left\{f_{k}\right\}_{k=1}^{\infty}$ be a Hausdorff frame for $\mathcal{H}$. The dual Hausdorff frames of $\left\{f_{k}\right\}_{k=1}^{\infty}$ are precisely the families $\left\{g_{n}\right\}_{n=1}^{\infty}$ where

$$
\begin{aligned}
g_{n}=\mathrm{S}^{-1} f_{n}+ & h_{n} \\
& -\sum_{j=1}^{\infty}\left\langle\sum_{k=1}^{n} \int_{0}^{1}\left(\begin{array}{c}
n-1 \\
k-1
\end{array}\right)(1-\alpha)^{n-k} \alpha^{k-1} d \mu(\alpha) S^{-1} f_{k}, \sum_{k=1}^{j} \int_{0}^{1}\left(\begin{array}{c}
j-1 \\
k-1
\end{array}\right)(1-\alpha)^{j-k} \alpha^{k-1} d \mu(\alpha) f_{k}\right\rangle h_{j}
\end{aligned}
$$

and $\left\{h_{k}\right\}_{k=1}^{\infty}$ is a Hausdorff Bessel sequence in $\mathcal{H}$.

3.4. Euler Frames and bounded below matrix mapping from $b v_{p}$ into $e_{w, p}^{\theta}$. For $0<p<\infty$, the Euler weighted sequence space $e_{w, p}^{\theta}$ defined by

$$
e_{w, p}^{\theta}=\left\{\left(x_{n}\right): \sum_{n=1}^{\infty} w_{n}\left|\sum_{k=1}^{n}\left(\begin{array}{c}
n-1 \\
k-1
\end{array}\right)(1-\theta)^{n-k} \theta^{k-1} x_{k}\right|^{p}<\infty\right\},
$$

where $0<\theta<1$ and $w=\left(w_{n}\right)$ is an increasing non-negative sequence of real numbers. More precisely, $e_{w, p}^{\theta}$ is the space of all sequences whose $E(\theta)$ transforms are in the space $\ell_{p}(w)$, where $\ell_{p}(w)$ denote the space of all complexvalued sequences $x=\left(x_{n}\right)$ for which

$$
\|x\|_{\ell_{p}(w)}:=\left(\sum w_{n}\left|x_{n}\right|^{p}\right)^{1 / p}<\infty
$$

and $\mathrm{E}(\theta)=\left(e_{n, k}(\theta)\right)_{n, k \geq 1}$ denotes the Euler matrix defined by

$$
e_{n, k}(\theta)=\left\{\begin{array}{cl}
\left(\begin{array}{c}
n-1 \\
k-1
\end{array}\right)(1-\theta)^{n-k} \theta^{k-1} & 1 \leq k \leq n, \\
0 & k>n,
\end{array}\right.
$$


and the matrix form of $\mathrm{E}(\theta)$ is

$$
E(\theta)=\left(\begin{array}{ccccc}
1 & 0 & 0 & 0 & \cdots \\
(1-\theta) & \theta & 0 & 0 & \cdots \\
(1-\theta)^{2} & 2 \theta(1-\theta) & \theta^{2} & 0 & \cdots \\
(1-\theta)^{3} & 3 \theta(1-\theta)^{2} & 3 \theta^{2}(1-\theta) & \theta^{3} & \cdots \\
\vdots & \vdots & \vdots & \vdots & \ddots
\end{array}\right) .
$$

This matrix is a special case of Hausdorff matrices in which $d \mu(\alpha)$ be the point evaluation at $\alpha=\theta$.

Lashkaripour and the first author, in [20], studied the properties of the Euler weighted sequence space. They proved that $e_{w, p}^{\theta}$ is linearly isomorphic to the space $\ell_{p}(w)$, and that $e_{w, p}^{\theta}(0<p<1)$ is a $p$-normed space with the $p$-norm defined by \|\|$x\|\|:=\|x\|_{e_{w, p}^{\theta}}^{p}$ where

$$
\|x\|_{e_{w, p}^{\theta}}=\left(\sum_{n=1}^{\infty} w_{n}\left|\sum_{k=1}^{n}\left(\begin{array}{c}
n-1 \\
k-1
\end{array}\right)(1-\theta)^{n-k} \theta^{k} x_{k}\right|^{p}\right)^{\frac{1}{p}} .
$$

Moreover, they showed that if $0<p<1$, then

$$
\|x\|_{\ell_{p}(w)} \leq \theta^{1 / p}\|x\|_{e_{w, p}^{\theta}},
$$

which implies that $e_{w, p}^{\theta} \subseteq \ell_{p}(w)$.

In this section, we first consider the lower bound problem of some matrix mapping from the space $b v_{p}$ into the Euler weighted sequence space $e_{w, p}^{\theta}$. The lower bound involved here is the number $L_{b v_{p}, e_{w, q}^{\theta}}\left(H_{\mu}\right)$, which is defined as the supremum of those $l$, satisfying the following inequality

$$
\left\|H_{\mu} x\right\|_{e_{w, q}^{\theta}} \geq l\|x\|_{b v_{p}}
$$

where $x \geq 0, x \in b v_{p}, H_{\mu}$ is the Hausdorff matrix which is considered as a matrix mapping from the sequence space $b v_{p}$ into the Euler weighted sequence space $e_{w, q}^{\theta}$ and $0<q \leq p<1$. Our result provides a Hardy type formula as a lower estimate for $L_{b v_{p}, e_{w, p}^{\theta}}\left(H_{\mu}\right)$. Here the weight sequence $w$ is considered to be a monotone increasing one with $w_{n} \geq 1$ for all $n \geq 1$. In particular, we apply our results to some spacial Hausdorff matrices such as Cesàro, Hölder and Gamma matrices.

To prove our main result we need the following lemma, which is a generalization of ([4], Proposition 7.4).

Lemma 3.13. Let $0<q \leq p<1, \frac{1}{q}+\frac{1}{q^{*}}=1$ and suppose that $A$ is a lower triangular matrix with non-negative entries. If

$$
\sup _{n \geq 1} \sum_{k=1}^{n} a_{n, k}=R>0 \quad \& \quad \inf _{k \geq 1} \sum_{n=k}^{\infty} a_{n, k}=C,
$$

then

$$
L_{b v_{p}, e_{w, q}^{\theta}}(A) \geq \frac{\theta^{-1 / q} R^{1 / q^{*}} C^{1 / q}}{2} .
$$


Proof. Applying Hölder's inequality, we have

$$
\begin{aligned}
\sum_{k=1}^{n} a_{n, k} w_{k} x_{k}^{q} & =\sum_{k=1}^{n} a_{n, k}^{1-q}\left(a_{n, k} w_{k}^{1 / q} x_{k}\right)^{q} \\
& \leq\left(\sum_{k=1}^{n} a_{n, k}\right)^{1-q}\left(\sum_{k=1}^{n} a_{n, k} w_{k}^{1 / q} x_{k}\right)^{q} \\
& \leq R^{1-q}\left(\sum_{k=1}^{n} a_{n, k} w_{k}^{1 / q} x_{k}\right)^{q} .
\end{aligned}
$$

Since $w$ is increasing and $w_{n} \geq 1$, we have

$$
\begin{aligned}
& R^{1-q} \sum_{n=1}^{\infty} w_{n}\left(\sum_{k=1}^{\infty} a_{n, k} x_{k}\right)^{q}=R^{1-q} \sum_{n=1}^{\infty} w_{n}\left(\sum_{k=1}^{n} a_{n, k} x_{k}\right)^{q} \\
& \geq R^{1-q} \sum_{n=1}^{\infty}\left(\sum_{k=1}^{n} a_{n, k} w_{k}^{1 / q} x_{k}\right)^{q} \geq \sum_{n=1}^{\infty}\left(\sum_{k=1}^{n} a_{n, k} w_{k} x_{k}^{q}\right) \\
& =\sum_{k=1}^{\infty} w_{k} x_{k}^{q}\left(\sum_{n=k}^{\infty} a_{n, k}\right) \geq C \sum_{k=1}^{\infty} w_{k} x_{k}^{q} \geq C \sum_{k=1}^{\infty} w_{k} x_{k}^{p} \geq C \sum_{k=1}^{\infty} x_{k}^{p},
\end{aligned}
$$

which implies

$$
\|A x\|_{\ell^{q}(w)} \geq R^{1 / q *} C^{1 / q}\|x\|_{\ell^{p}} \geq \frac{R^{1 / q *} C^{1 / q}}{2}\|x\|_{b v_{p}}
$$

On the other hand, using (3.4), we have

$$
\|A x\|_{e_{w, q}^{\theta}} \geq(1 / \theta)^{1 / q}\|A x\|_{\ell^{q}(w)} .
$$

Hence $\|A x\|_{e_{w, q}^{\theta}} \geq \frac{\theta^{-1 / q} R^{1 / q^{*}} C^{1 / q}}{2}\|x\|_{b v_{p}}$, and so we have the desired conclusion. This completes the proof.

Lemma 3.14. Let $0<q \leq p \leq 1$ and $E(\beta)$ be the Euler matrix of order $\beta$. Then

$$
L_{b v_{p}, e_{w, q}^{\theta}}(E(\beta)) \geq \frac{\theta^{-1 / q} \beta^{-1 / q}}{2}, \quad(0<\beta<1) .
$$

Proof. If $A=E(\beta)$, then the column sums $A$ are all $1 / \beta$ and row sums of all 1. Applying Lemma 3.13 to the case $C=1 / \beta$ and $R=1$, we deduce that $L_{b v_{p}, e_{w, q}^{\theta}}(E(\beta)) \geq \frac{1}{2} \theta^{-1 / q} \beta^{-1 / q}$, for $0<q \leq p<1$. For the case $p=1(q \leq p)$, it follows from (3.4) and Fubini's theorem with monotonicity of the weighted 
sequence $w$ that

$$
\begin{aligned}
\|E(\beta) x\|_{e_{w, q}^{\theta}} & \geq \theta^{-1 / q}\|E(\beta) x\|_{\ell^{q}(w)} \\
& \geq \theta^{-1 / q}\|E(\beta) x\|_{\ell^{1}(w)} \\
& =\theta^{-1 / q} \sum_{n=1}^{\infty} w_{n}\left(\sum_{k=1}^{\infty} e_{n, k}(\beta) x_{k}\right) \\
& \geq \theta^{-1 / q} \sum_{k=1}^{\infty} w_{k}\left(\sum_{n=1}^{\infty} e_{n, k}(\beta)\right) x_{k} \\
& \geq \theta^{-1 / q} \beta^{-1} \sum_{k=1}^{\infty} w_{k} x_{k} \geq \theta^{-1 / q} \beta^{-1} \sum_{k=1}^{\infty} x_{k} \\
& \geq \frac{\theta^{-1 / q} \beta^{-1 / q}}{2}\|x\|_{b v_{p}}(x \geq 0),
\end{aligned}
$$

which gives the desired inequality. This completes the proof.

Lemma 3.14 generalizes Lemma 2.2 in [10].

For $x \geq 0$, we have $H_{\mu} x=\int_{0}^{1} E(\alpha) x d \mu(\alpha)$. Hence Lemma 3.14 enables us to estimate the value of $L_{b v_{p}, e_{w, q}^{\theta}}\left(H_{\mu}\right)$.

Theorem 3.15. Let $0<q \leq p \leq 1$, and $q^{*}$ be the conjugate exponent of $q$, i.e. $1 / q+1 / q^{*}=1$. If $H_{\mu}$ be the Hausdorff matrix, then

$$
b v_{p}, e_{w, q}^{\theta}\left(H_{\mu}\right) \geq \theta^{-1 / q} \int_{0}^{1} \alpha^{-1 / q} d \mu(\alpha) .
$$

Proof. Consider (3.5), let $x \geq 0$ and $\|x\|_{b v_{p}}=1$. Applying Minkowski's inequality and Lemma 3.14 we obtain

$$
\begin{aligned}
\left\|H_{\mu} x\right\|_{e_{w, q}^{\theta}} & =\left\|\int_{0}^{1} E(\alpha) x d \mu(\alpha)\right\|_{e_{w, q}^{\theta}} \\
& \geq \int_{(0,1]}\|E(\alpha) x\|_{e_{w, q}^{\theta}} d \mu(\alpha) \\
& \geq\left(\theta^{-1 / q} \int_{(0,1]} \alpha^{-1 / q} d \mu(\alpha)\right)\|x\|_{b v_{p}} \\
& =\theta^{-1 / q} \int_{(0,1]} \alpha^{-1 / q} d \mu(\alpha) .
\end{aligned}
$$

This leads us to (3.5) and completes the proof.

Theorem 3.15 generalizes Theorem 2.3 in [10].

In the following, we present several special case of Theorem 3.15. Let $d \mu(\alpha)=$ $\beta(1-\alpha)^{\beta-1} d \alpha$, where $\beta>0$. Then $H_{\mu}$ reduces to the Cesàro matrix $C(\beta)$ (see [5], p.410). For $0<q \leq 1$, we have

$$
\int_{(0,1]} \alpha^{-1 / q} d \mu(\alpha)=\beta \int_{(0,1]} \alpha^{-1 / q}(1-\alpha)^{\beta-1} d \alpha=\infty .
$$


Applying (3.5), we get the following result.

Corollary 3.16. Let $\beta>0$. Then $L_{b v_{p}, e_{w, q}^{\theta}}(C(\beta))=\infty$ for $0<q \leq p \leq 1$.

Next, consider the case $d \mu(\alpha)=\frac{|\log \alpha|^{\beta-1}}{\Gamma(\beta)} d \alpha$, where $\beta>0$. For this case, $H_{\mu}$ reduces to the Hölder matrix $H(\beta)$ (see [5], p.410). We have

$$
\int_{(0,1]} \alpha^{-1 / q} d \mu(\alpha)=\infty, \quad(0<q \leq 1)
$$

Hence, the following is a consequence of (3.5).

Corollary 3.17. Let $\beta>0$. Then $L_{b v_{p}, e_{w, q}^{\theta}}(H(\beta))=\infty$ for $0<q \leq p \leq 1$.

The third special case that we consider is $d \mu(\alpha)=\beta \alpha^{\beta-1} d \alpha$, where $\beta>0$. Then $H_{\mu}$ becomes the Gamma matrix $\Gamma(\beta)$ (see [5], p.410). We have

$$
\int_{(0,1]} \alpha^{-1 / q} d \mu(\alpha)=\beta \int_{(0,1]} \alpha^{-1 / q+\beta-1} d \alpha= \begin{cases}\infty & \beta \leq 1 / q \\ \frac{\beta}{\beta-1 / q} & \beta>1 / q .\end{cases}
$$

Applying Theorem 3.15, we get the following corollary.

Corollary 3.18. Let $\alpha>0$ and $0<q \leq p \leq 1$. Then $L_{b v_{p}, e_{w, q}^{\theta}}(\Gamma(\beta))=\infty$, for $\beta \leq 1 / q$. Also, $L_{b v_{p}, e_{w, q}^{\theta}}(\Gamma(\beta)) \geq \theta^{-1 / q} \frac{\beta}{\beta-1 / q}$, for $\beta>1 / q$.

By definition an Euler frame of order $\theta$ or an $\mathrm{E}(\theta)$-frame for $\mathcal{H}$ is a sequence $\left\{f_{k}\right\}_{k=1}^{\infty}$ for which there exist positive real numbers $A$ and $B$ such that

$$
A\|f\|^{2} \leq\left\|\left\{\left\langle f, \sum_{k=1}^{n}\left(\begin{array}{c}
n-1 \\
k-1
\end{array}\right)(1-\theta)^{n-k} \theta^{k-1} f_{k}\right\rangle\right\}_{n=1}^{\infty}\right\|_{\ell^{2}}^{2} \leq B\|f\|^{2}, \quad \forall f \in \mathcal{H} .
$$

By the above notation we can modify the definition of an Euler frame of order $\theta$ for $\mathcal{H}$ as a sequence $\left\{f_{k}\right\}_{k=1}^{\infty}$ for which there exist positive real numbers $A$ and $B$ such that

$$
A\|f\|^{2} \leq\left\|\left\{\left\langle f, f_{k}\right\rangle\right\}_{k=1}^{\infty}\right\|_{e_{2}^{\theta}}^{2} \leq B\|f\|^{2}, \quad \forall f \in H
$$

where $e_{2}^{\theta}$ is the Euler weighted sequence space in which the weighted sequence $w=(1,1, \cdots)$. We observe that the Euler frames are related to the Euler space $e_{2}^{\theta}$.

Theorem 3.19. Let $\left\{f_{k}\right\}_{k=1}^{\infty}$ be a Bessel sequence with the Bessel bound B. Then it is an Euler Bessel sequence of order $\theta$ with the Euler Bessel bound B/O. 
Proof. Let $f \in \mathcal{H}$. By Hölder's inequality we have

$$
\begin{aligned}
\left\|\left\{\left\langle f, f_{k}\right\rangle\right\}_{k=1}^{\infty}\right\|_{e_{2}^{\theta}}^{2}=\sum_{n=1}^{\infty}\left|\sum_{k=1}^{n}\left(\begin{array}{c}
n-1 \\
k-1
\end{array}\right)(1-\theta)^{n-k} \theta^{k-1}\left\langle f, f_{k}\right\rangle\right|^{2} \\
\leq \sum_{n=1}^{\infty}\left\{\sum_{k=1}^{n}\left(\begin{array}{c}
n-1 \\
k-1
\end{array}\right)(1-\theta)^{n-k} \theta^{k-1}\left|\left\langle f, f_{k}\right\rangle\right|^{2}\right\} \underbrace{\left\{\sum_{k=1}^{n}\left(\begin{array}{c}
n-1 \\
k-1
\end{array}\right)(1-\theta)^{n-k} \theta^{k-1}\right\}}_{=1} \\
\leq \sum_{n=1}^{\infty} \sum_{k=1}^{n}\left(\begin{array}{c}
n-1 \\
k-1
\end{array}\right)(1-\theta)^{n-k} \theta^{k-1}\left|\left\langle f, f_{k}\right\rangle\right|^{2} \\
\leq \sum_{k=1}^{\infty}\left|\left\langle f, f_{k}\right\rangle\right|^{2} \sum_{n=k}^{\infty}\left(\begin{array}{c}
n-1 \\
k-1
\end{array}\right)(1-\theta)^{n-k} \theta^{k-1} \\
\leq\left(\frac{1}{\theta}\right) \sum_{k=1}^{\infty}\left|\left\langle f, f_{k}\right\rangle\right|^{2} \leq\left(\frac{B}{\theta}\right)\|f\|^{2},
\end{aligned}
$$

which implies that $\left\{f_{k}\right\}_{k=1}^{\infty}$ is an Euler Bessel sequence of order $\theta$ with the Euler Bessel bound $B / \theta$.

The converse of Theorem 3.19 is not true in general. That is, there exists an Euler Bessel sequence of order $\theta(0<\theta<1)$, which is not a Bessel sequence. To show this, let $\left\{e_{k}\right\}_{k=1}^{\infty}$ be an orthonormal basis for $\mathcal{H}$. Then the sequence $\left\{f_{n}(\theta)\right\}_{n=1}^{\infty}$ defined by

$$
f_{n}(\theta):=\sum_{k=1}^{n}\left(\begin{array}{c}
n-1 \\
k-1
\end{array}\right)(\theta-1)^{n-k} \theta^{1-n} e_{k} \quad(n=1,2, \ldots),
$$

is an Euler Bessel sequence of order $\theta$ with the Euler Bessel bound 1, but it is not a Bessel sequence in general. Indeed, let $f=e_{1}$, then we have

$$
\begin{aligned}
\sum_{n=1}^{\infty}\left|\left\langle f, f_{n}(\theta)\right\rangle\right|^{2} & =\sum_{n=1}^{\infty}\left|\left\langle e_{1}, \sum_{k=1}^{n}\left(\begin{array}{c}
n-1 \\
k-1
\end{array}\right)(\theta-1)^{n-k} \theta^{1-n} e_{k}\right\rangle\right|^{2} \\
& =1+\left(1-\frac{1}{\theta}\right)^{2}+\left(1-\frac{1}{\theta}\right)^{4}+\left(1-\frac{1}{\theta}\right)^{6}+\cdots \\
& =\sum_{n=1}^{\infty}\left\{\left(1-\frac{1}{\theta}\right)^{2}\right\}^{n-1}= \begin{cases}\infty & 0<\theta<\frac{1}{2}, \\
\frac{\theta^{2}}{2 \theta-1} & \frac{1}{2}<\theta<1,\end{cases}
\end{aligned}
$$

which implies that $\left\{f_{n}(\theta)\right\}_{n=1}^{\infty}$ is not a Bessel sequence whenever $0<\theta<1 / 2$. This sequence is also an Euler orthonormal basis for $\mathcal{H}$ and therefore is an example of Euler frame which is not a frame whenever $0<\theta<1 / 2$.

Proposition 3.20. Let $\left\{f_{k}\right\}_{k=1}^{\infty}$ be an Euler frame of order $\theta$ for $\mathcal{H}$ with the lower Euler frame bound $A$. Then it satisfies the lower frame condition with the lower frame bound $A \theta$.

Proof. The proof is a consequence of the following inequalities

$$
A\|f\|^{2} \leq\left\|\left\{\left\langle f, f_{k}\right\rangle\right\}_{k=1}^{\infty}\right\|_{e_{2}^{\theta}}^{2} \leq\left(\frac{1}{\theta}\right)\left\|\left\{\left\langle f, f_{k}\right\rangle\right\}_{k=1}^{\infty}\right\|_{\ell^{2}}^{2} .
$$


Theorem 3.21. Let $\left\{f_{k}\right\}_{k=1}^{\infty}$ be an Euler Bessel sequence of order $\theta$ with the Bessel bound $B$, and $0<\lambda \leq \theta<1$. Then $\left\{f_{k}\right\}_{k=1}^{\infty}$ is an Euler Bessel sequence of order $\lambda$ with the Euler Bessel bound $B \theta / \lambda$.

Proof. Since $\left\{f_{k}\right\}_{k=1}^{\infty}$ is an Euler Bessel sequence of order $\theta$ with the Euler Bessel bound $B$, we have

$$
\left\|\left\{\left\langle f, f_{k}\right\rangle\right\}_{k=1}^{\infty}\right\|_{e_{2}^{\theta}}^{2} \leq B\|f\|^{2}, \forall f \in \mathcal{H}
$$

Moreover, by the same reason as we have seen in Lemma 2.4 of [22], for $0<\lambda \leq$ $\theta<1$ we deduce

$$
\left\|\left\{\left\langle f, f_{k}\right\rangle\right\}_{k=1}^{\infty}\right\|_{e_{2}^{\lambda}} \leq\left(\frac{\theta}{\lambda}\right)^{\frac{1}{2}}\left\|\left\{\left\langle f, f_{k}\right\rangle\right\}_{k=1}^{\infty}\right\|_{e_{2}^{\theta}} .
$$

Putting (3.6) and (3.7) together, we get

$$
\left\|\left\{\left\langle f, f_{k}\right\rangle\right\}_{k=1}^{\infty}\right\|_{e_{2}^{\lambda}}^{2} \leq\left(\frac{B \theta}{\lambda}\right)\|f\|^{2}, \quad \forall f \in \mathcal{H}
$$

This implies that $\left\{f_{k}\right\}_{k=1}^{\infty}$ is an Euler Bessel sequence of order $\lambda$ with the Euler Bessel bound $B \theta \lambda^{-1}$.

We have the following characterization for Euler orthonormal bases of order $\theta$, Euler Riesz bases of order $\theta$ and Euler frames of order $\theta$ :

Corollary 3.22. Let $\left\{e_{k}\right\}_{k=1}^{\infty}$ be an orthonormal basis for $\mathcal{H}$. Then

(i) The Euler orthonormal bases of order $\theta$ for $\mathcal{H}$ are precisely the families

$$
\left\{U\left(\sum_{k=1}^{n}\left(\begin{array}{l}
n-1 \\
k-1
\end{array}\right)(\theta-1)^{n-k} \theta^{1-n} e_{k}\right)\right\}_{n=1}^{\infty},
$$

where $U$ is an unitary operator on $\mathcal{H}$.

(ii) An Euler Riesz basis of order $\theta$ is a family of the form

$$
\left\{U\left(\sum_{k=1}^{n}\left(\begin{array}{c}
n-1 \\
k-1
\end{array}\right)(\theta-1)^{n-k} \theta^{1-n} e_{k}\right)\right\}_{n=1}^{\infty},
$$

where $U$ is a bounded bijective operator on $\mathcal{H}$.

(iii) The Euler frames of order $\theta$ are precisely the families

$$
\left\{U\left(\sum_{k=1}^{n}\left(\begin{array}{l}
n-1 \\
k-1
\end{array}\right)(\theta-1)^{n-k} \theta^{1-n} e_{k}\right)\right\}_{n=1}^{\infty},
$$

where $\mathrm{U}$ is bounded and surjective operator on $\mathcal{H}$. 
Corollary 3.23. Let $\left\{f_{k}\right\}_{k=1}^{\infty}$ be an Euler frame of order $\theta$ for $\mathcal{H}$. The dual Euler frames of order $\theta$ for $\left\{f_{k}\right\}_{k=1}^{\infty}$ are precisely the families

$$
\left\{g_{n}\right\}_{n=1}^{\infty}=\left\{\mathrm{S}^{-1} f_{n}+h_{n}-\sum_{j=1}^{\infty}\left\langle\sum_{k=1}^{n}\left(\begin{array}{c}
n-1 \\
k-1
\end{array}\right)(1-\theta)^{n-k} \theta^{k-1} S^{-1} f_{k}, \sum_{k=1}^{j}\left(\begin{array}{c}
j-1 \\
k-1
\end{array}\right)(1-\theta)^{j-k} \theta^{k-1} f_{k}\right\rangle h_{j}\right\}_{n=1}^{\infty},
$$

where $\left\{h_{k}\right\}_{k=1}^{\infty}$ is an Euler Bessel sequence of order $\theta$ in $\mathcal{H}$.

Acknowledgement. The authors have beneted a lot from the referees report. So, we thank the reviewer for his/her careful reading and making some useful comments which improved the presentation of the paper. Also the first author would like to record his pleasure to Shahid Sayyid Gholamreza Mirafzali and Shahid Sayyid Mohammad Reza Mirafzali for their spiritual support.

\section{REFERENCES}

1. A. Aldroubi, Q. Sung and W. Tang, $p$-frames and shift invariant subspaces of $L^{p}$, J. Fourier Anal. Appl. 7 (2001), 1-22.

2. B. Altay, F. Basarar and M. Mursaleen, On the Euler sequence spaces which include the spaces $\ell^{p}$ and $\ell^{\infty} I$, Inform. Sci. 176 (2002), 1450-1462.

3. F. Basarr and B. Altay, On the space of sequences of p-bounded variation and related matrix mappings, Ukrainian Math. J. 55 (2003), no. 1, $136-147$.

4. G. Bennett, Factorizing the classical inequalities, Mem. Amer. Math. Soc. 120 (1996), no. $576,1-130$.

5. G. Bennett, Inequalities complimentary to Hardy, Quart. J. Math. Oxford, Ser. 49 (1998), no. 4, 395-432.

6. P.G. Casazza, The art of frame theory, Taiwang J. Math. 4 (2000), no. 2, 129-201.

7. P.G. Casazza and O. Christensen, D. Stoeva, Frame expansions in separable Banach spaces, J. Math. Anal. Appl. 1 (2005), 1-14.

8. H.-X. Cao, Bessel sequences in a Hilbert space, Gongcheng Shuxue Xuebao 17 (2000), no. 2, 92-98.

9. C.-P. Chen and K.-Z. Wang, Lower bounds of Copson type for the transposes of lower triangular matrieces, J. Math. Anal. Appl. 341 (2008), 1284-1294.

10. C.-P. Chen and K.-Z. Wang, Lower bounds of Copson type for Hausdorff matrices II, Linear Algebra Appl. 422 (2007), 563-573.

11. O. Christensen, Frames and Riesz bases An Introductory Course, Birkhäuser, Boston, Basel, Berlin, 2007.

12. J.B. Conway, A Course in Functional Analysis, 2nd Edition, Springer-Verlag, 1990.

13. I. Daubechies, A. Grossmann and Y. Meyer, Painless nonorthogonal expansion, J. Math. Phys. 27 (1986), 1271-1283.

14. M.A. Dehghan and M.A. Hasankhani Fard, g-dual frames in Hilbert spaces, U.P.B. Sci. Bull., Series A, 75 (2013), no. 1, 129-140.

15. R.J. Duffin and A C. Schaeffer, A class of nonharmonic Fourier series, Trans. Amer. Math. Soc. 72 (1952), 341-366.

16. K. Grochenig, Describing functions: atomic decompositions versus frames, Monatsh. Math. 112 (1991), 1-41.

17. D. Han and D. Larson, Frame, bases and group representations, Memoir. Amer. Math. Soc. 147 (2000), 1-94.

18. C.-Y. Li and H.-X. Cao, $X_{d}$ frames and Riesz bases for a Banach space (Chinese) Acta Math. Sinica (Chin. Ser.) 49 (2006), no. 6, 1361-1366.

19. R. Lashkaripour and D. Foroutannia, Some inequalities involving upper bounds for some matrix operators I, Czech. Math. J. 57 (2007), no. 2, 553-572. 
20. R. Lashkaripour and G. Talebi, Lower bound for matrix operators on the Euler weighted sequence space $e_{w, p}^{\theta}(0<p<1)$, Rend. Circ. Mat. Palermo 61 (2012), no. 1, 1-12.

21. R. Lashkaripour and G. Talebi, Lower bounds of Copson type for the transpose of matrices on weighted sequence spaces, Bull. Iranian Math. Soc. 37 (2011), no. 1, 115-126.

22. G. Talebi and M.A. Dehghan, Upper bounds for the operator norms of Hausdorff matrices and Nörlund matrices on the Euler weighted sequence space, Linear Multilinear Algebra 62 (2014), no. 10, 1275-1284.

23. R. Young, An introduction to nonharmonic Fourier series, Academic Press, New York, 1980 .

Department of Mathematics, Vali-e-Asr University of Rafsanjan, Rafsanjan, ISLAMIC REPUBLIC OF IRAN.

E-mail address: Gh.talebi@vru.ac.ir

E-mail address: Dehghan@vru.ac.ir 